\title{
Bohr-Jessen process and functional limit theorem
}

\author{
Satoshi Takanobu
}

\begin{abstract}
The Bohr-Jessen limit theorem states that for each $\sigma>\frac{1}{2}$, there exists an asymptotic probability distribution of $\log \zeta(\sigma+\sqrt{-1} \cdot)$. Here $\zeta(\cdot)$ is the Riemann zeta function, and $\log \zeta(\cdot)$ is a primitive function of $\zeta^{\prime} / \zeta$ on some simply connected domain of $\mathbb{C}$. In this paper, we generalize this limit theorem to a functional limit theorem and show a similar limit theorem for a continuous process $\{\log \zeta(\sigma+\sqrt{-1} \cdot)\}_{\sigma>1 / 2}$, which we call the Bohr--Jessen functional limit theorem.
\end{abstract}

\section{Introduction}

The Riemann zeta function $\zeta(\cdot)$ is defined on the half-plane $\{s \in \mathbb{C} ; \operatorname{Re} s>1\}$ by the Dirichlet series

$$
\zeta(s)=\sum_{n=1}^{\infty} \frac{1}{n^{s}},
$$

where $n^{s}=e^{s \log n}$. This function is holomorphic there and is also expressed by the Euler product

$$
\zeta(s)=\prod_{p: \text { prime }} \frac{1}{1-1 / p^{s}} .
$$

Moreover, $\zeta(\cdot)$ is analytically continuable to a meromorphic function on the whole complex plane $\mathbb{C}$ that is holomorphic except $s=1$ and has a simple pole at $s=1$ with residue 1 . Let us denote this meromorphic function by the same $\zeta(\cdot)$.

About zeros of $\zeta(\cdot)$, it is well known that

$$
\begin{aligned}
& \{\text { zeros of } \zeta(\cdot)\} \cap\{s \in \mathbb{C} ; \operatorname{Re} s \geq 1\}=\emptyset, \\
& \{\text { zeros of } \zeta(\cdot)\} \cap\{s \in \mathbb{C} ; \operatorname{Re} s \leq 0\}=\{-2,-4,-6, \ldots\},
\end{aligned}
$$

and it is conjectured that

$$
\{\text { zeros of } \zeta(\cdot)\} \cap\{s \in \mathbb{C} ; 0<\operatorname{Re} s<1\} \subset\left\{s \in \mathbb{C} ; \operatorname{Re} s=\frac{1}{2}\right\} .
$$

This is well known as the Riemann hypothesis and remains unsolved. Since $\zeta(s) \neq 0$ on $\left\{s \in \mathbb{C} ; \operatorname{Re} s>\frac{1}{2}\right\}$ under this hypothesis, the $\log$ zeta function $\log \zeta(\cdot)$

Kyoto Journal of Mathematics, Vol. 54, No. 2 (2014), 401-426

DOI 10.1215/21562261-2642440, (C) 2014 by Kyoto University

Received July 5, 2012. Revised January 18, 2013. Accepted February 25, 2013.

2010 Mathematics Subject Classification: Primary 60F17; Secondary 11M06. 
can be defined as a holomorphic function on $G^{\prime}=\left\{s \in \mathbb{C} ; \operatorname{Re} s>\frac{1}{2}\right\} \backslash\left(\frac{1}{2}, 1\right]$ with the derivative $\zeta^{\prime}(s) / \zeta(s)$ and the value $\log \left(\pi^{2} / 6\right)$ at $s=2$. Unfortunately, the Riemann hypothesis is not yet proved to hold valid at the moment, so a modification of this definition is necessary. In fact, in place of $G^{\prime}$, we only have to take a simply connected domain $G$ of $\mathbb{C}$ such that $G$ contains $\{s \in \mathbb{C} ; \operatorname{Re} s>1\}$ but no zeros of $\zeta(\cdot)$.

When $\sigma>1$, it is easily seen from (1.1) that $|\zeta(\sigma+\sqrt{-1} t)| \leq \zeta(\sigma)(\forall t \in \mathbb{R})$. Thus, $\zeta(\sigma+\sqrt{-1} t)$ takes the value in the closed disc with center at origin and radius $\zeta(\sigma)$. Then how is the case when $\sigma \leq 1$ ? In the early 1910s, H. Bohr obtained many results about the behavior of $\zeta(\cdot)$ on the strip $\left\{s \in \mathbb{C} ; \frac{1}{2}<\right.$ $\operatorname{Re} s \leq 1\}$. Among them, the following is an answer of the question above (see Bohr [1]).

FACT 1.1

For $\frac{1}{2}<\sigma \leq 1$, the set $\{\log \zeta(\sigma+\sqrt{-1} t) ; t \in \mathbb{R}$ with $\sigma+\sqrt{-1} t \in G\}$ is dense in $\mathbb{C}$.

Indeed, since $\zeta(\sigma+\sqrt{-1} t)=e^{\log \zeta(\sigma+\sqrt{-1} t)}$, it is seen from this fact that the set $\{\zeta(\sigma+\sqrt{-1} t) ; t \in \mathbb{R}\}$ is dense in $\mathbb{C}$ (see Bohr and Courant [2]). According to whether $\sigma>1$ or $\sigma \leq 1$, the behavior of $\zeta(\sigma+\sqrt{-1} \cdot)$ changes drastically.

Bohr studied the value distribution of $\zeta(\cdot)$, which motivated him to develop the theory of almost periodic functions. It was in the 1930s that he, together with B. Jessen, arrived at the following result (see Bohr and Jessen [3]).

FACT 1.2

Let $R$ be any closed rectangle in $\mathbb{C}$ with edges parallel to the axes. Then, for each $\sigma \in\left(\frac{1}{2}, \infty\right)$,

$$
\lim _{T \rightarrow \infty} \frac{1}{2 T} \mu(t \in[-T, T] ; \sigma+\sqrt{-1} t \in G, \log \zeta(\sigma+\sqrt{-1} t) \in R)
$$

exists. Here $\mu$ is the 1-dimensional Lebesgue measure.

This is the original version of the Bohr--Jessen limit theorem. The current version is stated in terms of the convergence of probability distributions.

FACT 1.3

For $T>0$, let $U_{T}$ be a real-valued random variable uniformly distributed on $[-T, T]$, and for $\sigma \in\left(\frac{1}{2}, \infty\right)$, put a complex-valued random variable $\widetilde{X}_{T}(\sigma)$ as

$$
\widetilde{X}_{T}(\sigma):=\mathbf{1}_{G}\left(\sigma+\sqrt{-1} U_{T}\right) \log \zeta\left(\sigma+\sqrt{-1} U_{T}\right) .
$$

Then the distribution of $\widetilde{X}_{T}(\sigma)$ on $(\mathbb{C}, \mathcal{B}(\mathbb{C}))$ is weakly convergent as $T \rightarrow \infty$. Here $\mathcal{B}(\mathbb{C})$ is the Borel $\sigma$-algebra of $\mathbb{C}$.

This proof can be found in Jessen and Wintner [6], Borchsenius and Jessen [4], Laurinčikas [9], [10], and [11], Matsumoto [12] and [13], and so on. 
From Fact 1.3, it is natural to ask the following questions:

(a) What is its limit distribution?

(b) For $\frac{1}{2}<\sigma_{1}<\cdots<\sigma_{n}<\infty$, is the joint distribution of $\left(\widetilde{X}_{T}\left(\sigma_{1}\right), \ldots\right.$, $\left.\widetilde{X}_{T}\left(\sigma_{n}\right)\right)$ weakly convergent as $T \rightarrow \infty$ ?

(c) In general, how is the convergence of a stochastic process $\left\{\widetilde{X}_{T}(\sigma)\right\}_{\sigma>1 / 2}$ as $T \rightarrow \infty$ ?

In [18], a probability space $\left(\mathbb{R}^{\mathbb{B}}, \mathcal{B}_{K}\left(\mathbb{R}^{\mathbb{B}}\right), \mathbf{P}\right)$ of large volume was prepared (see Proposition 2.1), on this space a complex-valued random variable

$$
X(\sigma)=\sum_{p}-\log \left(1-\frac{e(-\log p)}{p^{\sigma}}\right)
$$

was defined (see Definition 2.1), and its distribution was shown to be the limit distribution. Here and hereafter, $\sum_{p}$ is the sum extended over all primes. Thus, the question (a) is solved!

In this paper, the questions (b) and (c) are considered. In Section 2, it is shown that a stochastic process $\{X(\sigma)\}_{\sigma>1 / 2}$ has a continuous modification $\{\widetilde{X}(\sigma)\}_{\sigma>1 / 2}$ (see Theorem 2.1). This complex continuous process is called the Bohr-Jessen process. In Section 4, it is shown that the distribution of a continuous process $\left\{\widetilde{X}_{T}(\sigma)\right\}_{\sigma>1 / 2}$ is weakly convergent to that of a continuous process $\{\tilde{X}(\sigma)\}_{\sigma>1 / 2}$ as $T \rightarrow \infty$ (see Theorem 4.1). This limit theorem is called the Bohr-Jessen functional limit theorem. In Section 3, to make up our argument, the path space $C\left(\left(\frac{1}{2}, \infty\right) \rightarrow \mathbb{C}\right)$ is viewed. Note that $\widetilde{X}_{T}(\cdot)$ and $\widetilde{X}(\cdot)$ are both $C\left(\left(\frac{1}{2}, \infty\right) \rightarrow \mathbb{C}\right)$-valued random variables.

Before closing this introduction, let us mention a related result of Laurinčikas [11]. As a simple consequence of Theorem 4.1, we have a limit theorem for $\zeta(\cdot)$ that the distribution of a continuous process $\left\{\zeta\left(\sigma+\sqrt{-1} U_{T}\right)\right\}_{\sigma>1 / 2}$ is weakly convergent to that of a continuous process $\left\{e^{\widetilde{X}(\sigma)}\right\}_{\sigma>1 / 2}$ as $T \rightarrow \infty$ (see Corollary 4.1). This is a weak version of [11, Theorem 5.1.8], which stated the weak convergence of the distribution of a holomorphic process $\left\{\zeta\left(s+\sqrt{-1} U_{T}\right)\right\}_{\operatorname{Re} s>1 / 2}$ as $T \rightarrow \infty$. This is originally due to B. Bagchi. Our target in this paper is not $\zeta(\cdot)$ itself but $\log \zeta(\cdot)$. In the definition of $\log \zeta(\cdot)$, zeros of $\zeta(\cdot)$ are troublesome. At the moment when the Riemann hypothesis is still unsolved, the defining domain of $\log \zeta(\cdot)$ does not always contain the half-plane $\left\{s \in \mathbb{C} ; \operatorname{Re} s>\frac{1}{2}\right\}$. So we must pay attention to zeros of $\zeta(\cdot)$ in taking its logarithm. To this end, we restrict ourselves to a real variable $\sigma>\frac{1}{2}$ in $\log \zeta\left(\cdot+\sqrt{-1} U_{T}\right)$. Also, for the same reason, it seems that our Theorem 4.1 does not follow from [11, Theorem 5.1.8] as a corollary.

We express our sincere thanks to the referee who pointed out that Theorem 4.1 is closely concerned with [11, Theorem 5.1.8] and recommended that we refer to the connection between them. 


\section{Bohr-Jessen process}

A continuous function $f: \mathbb{R} \rightarrow \mathbb{C}$ is said to be almost periodic if the following holds:

$$
\forall \varepsilon>0, \exists l=l(\varepsilon)>0 \quad \text { s.t. }\left\{\begin{array}{l}
\forall \alpha \in \mathbb{R}, \exists \tau \in(\alpha, \alpha+l) \\
\text { s.t. } \sup _{t \in \mathbb{R}}|f(t+\tau)-f(t)| \leq \varepsilon .
\end{array}\right.
$$

Let $\mathbb{B}$ be the set of all real-valued almost periodic functions. For $T>0$, let $P_{T}$ be a uniform distribution on $[-T, T]$, that is,

$$
P_{T}(E)=\frac{1}{2 T} \mu([-T, T] \cap E), \quad E \in \mathcal{B}(\mathbb{R}) .
$$

Here $\mathcal{B}(\mathbb{R})$ is the Borel $\sigma$-algebra of $\mathbb{R}$. For $f_{1}, \ldots, f_{n} \in \mathbb{B}$, let $P_{T}^{\left(f_{1}, \ldots, f_{n}\right)}$ be an image measure of $P_{T}$ by the continuous mapping $\mathbb{R} \ni t \mapsto\left(f_{1}(t), \ldots, f_{n}(t)\right) \in \mathbb{R}^{n}$. Then, for each $\xi=\left(\xi_{1}, \ldots, \xi_{n}\right) \in \mathbb{R}^{n}$, its characteristic function $\widehat{P_{T}^{\left(f_{1}, \ldots, f_{n}\right)}}(\xi)$ is convergent to the mean value $M\left(e^{\sqrt{-1} \sum_{i=1}^{n} \xi_{i} f_{i}}\right)$ of an almost periodic function $\mathbb{R} \ni t \mapsto e^{\sqrt{-1} \sum_{i=1}^{n} \xi_{i} f_{i}(t)} \in \mathbb{C}$ as $T \rightarrow \infty$. Since, by Bochner's theorem, $M\left(e^{\sqrt{-1} \sum_{i=1}^{n} \xi_{i} f_{i}}\right)$ is a characteristic function of a probability measure $\mathbf{P}^{\left(f_{1}, \ldots, f_{n}\right)}$ on $\left(\mathbb{R}^{n}, \mathcal{B}\left(\mathbb{R}^{n}\right)\right), P_{T}^{\left(f_{1}, \ldots, f_{n}\right)}$ is weakly convergent to this probability measure as $T \rightarrow \infty$. A family $\left\{\mathbf{P}^{\left(f_{1}, \ldots, f_{n}\right)} ; f_{1}, \ldots, f_{n} \in \mathbb{B}, n \in \mathbb{N}\right\}$ of finite-dimensional probability measures satisfies the consistency condition: for $1 \leq i \leq n+1, \pi_{i}: \mathbb{R}^{n+1} \rightarrow$ $\mathbb{R}^{n}$ being $\pi_{i}\left(x_{1}, \ldots, x_{n+1}\right)=\left(x_{1}, \ldots, x_{i-1}, x_{i+1}, \ldots, x_{n+1}\right)$,

$$
\mathbf{P}^{\left(f_{1}, \ldots, f_{n+1}\right)} \circ \pi_{i}^{-1}=\mathbf{P}^{\left(f_{1}, \ldots, f_{i-1}, f_{i+1}, \ldots, f_{n+1}\right)}, \quad \forall f_{1}, \ldots, \forall f_{n+1} \in \mathbb{B} .
$$

Therefore, applying Kolmogorov's extension theorem (see Kotani [8, Theorem 4.22]), we can obtain the following proposition.

\section{PROPOSITION 2.1}

For distinct $f_{1}, \ldots, f_{n} \in \mathbb{B}$, let $\pi_{\left(f_{1}, \ldots, f_{n}\right)}: \mathbb{R}^{\mathbb{B}} \rightarrow \mathbb{R}^{n}$ be

$$
\pi_{\left(f_{1}, \ldots, f_{n}\right)}\left(\left(x_{f}\right)_{f \in \mathbb{B}}\right)=\left(x_{f_{1}}, \ldots, x_{f_{n}}\right),
$$

and let $\mathcal{B}_{K}\left(\mathbb{R}^{\mathbb{B}}\right)^{\dagger}=\sigma\left(\pi_{f} ; f \in \mathbb{B}\right)$. Then there exists a unique probability measure $\mathbf{P}$ on a measurable space $\left(\mathbb{R}^{\mathbb{B}}, \mathcal{B}_{K}\left(\mathbb{R}^{\mathbb{B}}\right)\right)$ such that

$$
\left\{\begin{array}{l}
\forall n \in \mathbb{N} \text { and } \forall f_{1}, \ldots, \forall f_{n} \in \mathbb{B} \text { with } f_{i} \neq f_{j}(i \neq j), \\
\quad \mathbf{P} \circ \pi_{\left(f_{1}, \ldots, f_{n}\right)}^{-1}=\mathbf{P}^{\left(f_{1}, \ldots, f_{n}\right)} .
\end{array}\right.
$$

For $\lambda \in \mathbb{R} \backslash\{0\}$, let $e(\lambda): \mathbb{R}^{\mathbb{B}} \rightarrow \mathbb{C}$ be

$$
e(\lambda)=\pi_{\cos \lambda .}+\sqrt{-1} \pi_{\sin \lambda .},
$$

where $\cos \lambda$. and $\sin \lambda$. denote periodic functions $t \mapsto \cos \lambda t$ and $t \mapsto \sin \lambda t$, respectively. A complex-valued random variable $e(\lambda)$ is uniformly distributed on the

$\dagger^{\dagger} \mathcal{B}_{K}\left(\mathbb{R}^{\mathbb{B}}\right)$ is called the Kolmogorov $\sigma$-algebra on $\mathbb{R}^{\mathbb{B}}$. 
unit circle of $\mathbb{C}$; that is, $\forall f: \mathbb{C} \rightarrow \mathbb{C}$ that are bounded Borel measurable

$$
E^{\mathbf{P}}[f(e(\lambda))]=\frac{1}{2 \pi} \int_{0}^{2 \pi} f\left(e^{\sqrt{-1} t}\right) d t .
$$

Here and hereafter, $E^{\mathbf{P}}$ stands for expectation with respect to $\mathbf{P}$. If a real sequence $\left\{\lambda_{i}\right\}_{i=1}^{\infty}$ is linearly independent over $\mathbb{Q}$, a sequence of complex-valued random variables $\left\{e\left(\lambda_{i}\right)\right\}_{i=1}^{\infty}$ is independent and identically distributed (i.i.d.). When $\lambda_{i}=-\log p_{i}$, where $p_{i}$ is the $i$ th prime number, $\left\{-\log p_{i}\right\}_{i=1}^{\infty}$ is linearly independent over $\mathbb{Q}$ by the uniqueness of prime factorization. Then we have the following claim.

CLAIM 2.1

For $s \in \mathbb{C}, \operatorname{Re} s>\frac{1}{2}, \sum_{i=1}^{\infty}-\log \left(1-\left(e\left(-\log p_{i}\right)\right) / p_{i}^{s}\right)$ is convergent $\mathbf{P}$-almost everywhere. Here

$$
\log z:=\int_{1}^{z} \frac{d w}{w}, \quad z \in \mathbb{C} \backslash(-\infty, 0]
$$

\section{Proof}

Fix $s \in \mathbb{C}$, Re $s>\frac{1}{2}$. Since, for each prime $p,\left|e(-\log p) / p^{s}\right|=1 / p^{\operatorname{Re} s}<1 / \sqrt{2}<1$, and thus $1-e(-\log p) / p^{s} \in \mathbb{C} \backslash(-\infty, 0],-\log \left(1-e(-\log p) / p^{s}\right)$ is well defined. By (2.3),

$$
\begin{aligned}
E^{\mathbf{P}}\left[-\log \left(1-\frac{e(-\log p)}{p^{s}}\right)\right] \\
=\frac{1}{2 \pi} \int_{0}^{2 \pi}-\log \left(1-\frac{e^{\sqrt{-1} t}}{p^{s}}\right) d t \\
=\frac{1}{2 \pi \sqrt{-1}} \int_{|z|=1} \frac{-\log \left(1-z / p^{s}\right)}{z-0} d z \\
=-\log \left(1-\frac{0}{p^{s}}\right) \\
\quad\left[\odot-\log \left(1-z / p^{s}\right) \text { is holomorphic on }\{z \in \mathbb{C} ;|z|<\sqrt{2}\}\right] \\
=0 .
\end{aligned}
$$

Also, by $|-\log (1-z)| \leq|z| /(1-|z|)(|z|<1)$,

$$
E^{\mathbf{P}}\left[\left|-\log \left(1-\frac{e(-\log p)}{p^{s}}\right)\right|^{2}\right] \leq\left(\frac{1 / p^{\operatorname{Re} s}}{1-1 / p^{\operatorname{Re} s}}\right)^{2} \leq\left(\frac{2^{\operatorname{Re} s}}{2^{\operatorname{Re} s}-1}\right)^{2} \frac{1}{p^{2 \operatorname{Re} s}} .
$$

From the convergence $\sum_{p} 1 / p^{2} \operatorname{Re} s<\infty$ and the independence of $\{-\log (1-$ $\left.\left.e(-\log p) / p^{s}\right)\right\}_{p}$, we apply Proposition 2.2 below to have the assertion of Claim 2.1. 
DEFINITION 2.1

For simplicity, let $D=\left\{s \in \mathbb{C} ; \operatorname{Re} s>\frac{1}{2}\right\}$. For $s \in D$, put

$$
X(s):=\sum_{p}-\log \left(1-\frac{e(-\log p)}{p^{s}}\right) .
$$

\section{THEOREM 2.1}

We have that $\{X(s)\}_{s \in D}$ has a continuous modification. Namely,

$\exists \widetilde{X}: D \times \mathbb{R}^{\mathbb{B}} \rightarrow \mathbb{C}$

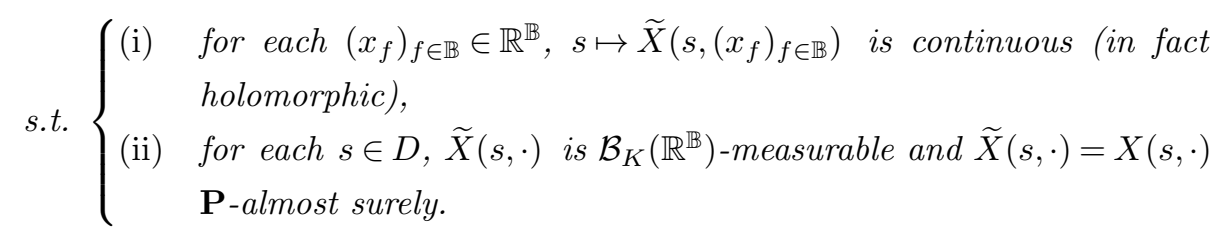

Proof

For $w \in \mathbb{C},|w|<1$, put

$$
R_{3}(w):=-\log (1-w)-w-\frac{w^{2}}{2} .
$$

Note that $\left|R_{3}(w)\right| \leq(1 / 3)\left[|w|^{3} /(1-|w|)\right] \quad(|w|<1)$, since $R_{3}(w)=w^{3} \int_{0}^{1}\left[t^{2} /\right.$ $(1-t w)] d t$. For each $s \in D$,

$$
\begin{aligned}
X(s) & =\sum_{p}-\log \left(1-\frac{e(-\log p)}{p^{s}}\right) \\
& =\sum_{p} \frac{e(-\log p)}{p^{s}}+\frac{1}{2} \sum_{p} \frac{e(-\log p)^{2}}{p^{2 s}}+\sum_{p} R_{3}\left(\frac{e(-\log p)}{p^{s}}\right) .
\end{aligned}
$$

Since

$$
\left|R_{3}\left(\frac{e(-\log p)}{p^{s}}\right)\right| \leq \frac{1}{3} \frac{1}{1-1 / p^{\operatorname{Re} s}} \frac{1}{p^{3 \operatorname{Re} s}},
$$

$\sum_{p} R_{3}\left(e(-\log p) / p^{s}\right)$ is absolutely convergent on $\left\{s \in \mathbb{C} ; \operatorname{Re} s>\frac{1}{3}\right\}$ and holomorphic there.

Then, $\{e(-\log p)\}_{p}$ and $\left\{e(-\log p)^{2}\right\}_{p}$ are independent and of mean zero, respectively. And, since

$$
\begin{gathered}
\sum_{p} E^{\mathbf{P}}\left[\left|\frac{e(-\log p)}{p^{s}}\right|^{2}\right]=\sum_{p} \frac{1}{p^{2 \operatorname{Re} s}}<\infty \quad \text { if } \operatorname{Re} s>\frac{1}{2}, \\
\sum_{p} E^{\mathbf{P}}\left[\left|\frac{e(-\log p)^{2}}{p^{2 s}}\right|^{2}\right]=\sum_{p} \frac{1}{p^{4 \operatorname{Re} s}}<\infty \quad \text { if } \operatorname{Re} s>\frac{1}{4},
\end{gathered}
$$

we apply Proposition 2.2 below to see that 


$$
\begin{aligned}
& \sum_{p} \frac{e(-\log p)}{p^{s}} \text { is convergent } \mathbf{P} \text {-a.e. } \quad \text { if } \operatorname{Re} s>\frac{1}{2}, \\
& \sum_{p} \frac{e(-\log p)^{2}}{p^{2 s}} \text { is convergent } \mathbf{P} \text {-a.e. } \quad \text { if } \operatorname{Re} s>\frac{1}{4} .
\end{aligned}
$$

Now let

$$
\Omega_{0}:=\bigcap_{n=1}^{\infty}\left\{\text { the series } \sum_{p} \frac{e(-\log p)}{p^{1 / 2+1 / n}} \text { is convergent }\right\} .
$$

From the above, $\mathbf{P}\left(\Omega_{0}\right)=1$. On $\Omega_{0}$, the series $\sum_{p} e(-\log p) / p^{1 / 2+1 / n}$ is convergent for each $n \in \mathbb{N}$, and thus, by Proposition 2.3 below, $\sum_{p} e(-\log p) / p^{s}$ is convergent on $D=\left\{s \in \mathbb{C} ; \operatorname{Re} s>\frac{1}{2}\right\}$ and holomorphic there. Also, since

$$
\sum_{p}\left|\frac{e(-\log p)^{2}}{p^{2 s}}\right|=\sum_{p} \frac{1}{p^{2 \operatorname{Re} s}}<\infty \quad(s \in D),
$$

$\sum_{p} e(-\log p)^{2} / p^{2 s}$ is absolutely convergent on $D$ and holomorphic there.

By putting all this together, the desired continuous (in fact, holomorphic) modification $\widetilde{X}$ is as follows:

$$
\widetilde{X}(s):= \begin{cases}\sum_{p}-\log \left(1-\frac{e(-\log p)}{p^{s}}\right) & \text { on } \Omega_{0}, \\ 0 & \text { on } \mathbb{R}^{\mathbb{B}} \backslash \Omega_{0} .\end{cases}
$$

\section{DEFINITION 2.2}

We call $\widetilde{X}$ the Bohr-Jessen process (in the wide sense).

In general, for a sequence $\left\{\Theta_{p}\right\}_{p \text { :prime }}$ of independent complex-valued random variables such that each $\Theta_{p}$ is uniformly distributed on the unit circle of $\mathbb{C}$, we call a continuous modification of $\left\{\sum_{p}-\log \left(1-\Theta_{p} / p^{s}\right)\right\}_{s \in D}$ the Bohr-Jessen process. The restriction of $\Theta_{p}=e(-\log p)$ is not necessary for this definition only. But, to make the argument in Section 4 clear, we do so in this paper.

We here gather two propositions cited in the proofs of Claim 2.1 and Theorem 2.1.

\section{PROPOSITION 2.2}

Let $\left\{X_{n}\right\}_{n=1}^{\infty}$ be a sequence of real-valued random variables defined on a probability space $(\Omega, \mathcal{F}, P)$. Suppose that

- $\left\{X_{n} ; n=1,2, \ldots\right\}$ are independent,

- $X_{n}$ is square integrable, that is, $E\left[X_{n}^{2}\right]<\infty(\forall n)$,

- $\sum_{n=1}^{\infty} \operatorname{Var}\left(X_{n}\right)<\infty$, where $\operatorname{Var}\left(X_{n}\right)$ is the variance of $X_{n}$, that is, $\operatorname{Var}\left(X_{n}\right)=E\left[\left(X_{n}-E\left[X_{n}\right]\right)^{2}\right]$.

Then $\sum_{n=1}^{\infty}\left(X_{n}-E\left[X_{n}\right]\right)$ is convergent P-almost everywhere; that is, $\sum_{n=1}^{N}\left(X_{n}-E\left[X_{n}\right]\right)$ is convergent as $N \rightarrow \infty$ P-almost everywhere. 
For the proof, see Itô [5, Theorem 4.2.1], Sato [16, Theorem 10.1], or Stroock [17, Theorem 1.4.2].

\section{PROPOSITION 2.3}

Let $\sigma \in \mathbb{R}$, and let $\left\{a_{n}\right\}_{n=1}^{\infty}$ be a complex sequence. Suppose that the series $\sum_{n=1}^{\infty} a_{n} / n^{\sigma}$ is convergent. Then, for $0<\theta<\frac{\pi}{2}$ the Dirichlet series $\sum_{n=1}^{\infty} a_{n} / n^{s}$ is uniformly convergent on $\{s \in \mathbb{C} ;|\arg (s-\sigma)| \leq \theta\}$. Thus, this Dirichlet series is convergent on $\{s \in \mathbb{C} ; \operatorname{Re} s>\sigma\}$ and holomorphic there.

For the proof, see Kawada [7, Section 6.1] or Tenenbaum [19, Chapter II.1, Theorem 3].

From [15], we know the central limit theorem

the distribution of $\frac{\widetilde{X}(\sigma)}{\sqrt{C_{\sigma}}}$

$\rightarrow$ the standard normal distribution of $\mathbb{C}$ as $\sigma \searrow \frac{1}{2}$,

where $C_{\sigma}:=\frac{1}{2} \sum_{p} 1 / p^{2 \sigma}$. Since, for each $A>0$,

$$
\begin{aligned}
\mathbf{P}(|\tilde{X}(\sigma)| \leq A) & =\mathbf{P}\left(\left|\frac{\tilde{X}(\sigma)}{\sqrt{C_{\sigma}}}\right| \leq \frac{A}{\sqrt{C_{\sigma}}}\right) \\
& \leq \mathbf{P}\left(\left|\frac{\tilde{X}(\sigma)}{\sqrt{C_{\sigma}}}\right| \leq \frac{A}{\sqrt{C_{\sigma_{0}}}}\right), \quad 1 / 2<\sigma \leq \sigma_{0},
\end{aligned}
$$

letting $\sigma \searrow \frac{1}{2}$ and then $\sigma_{0} \searrow \frac{1}{2}$ yields that

$$
\begin{aligned}
\limsup _{\sigma \searrow 1 / 2} \mathbf{P}(|\tilde{X}(\sigma)| \leq A) & \leq \limsup _{\sigma \searrow 1 / 2} \mathbf{P}\left(\left|\frac{\tilde{X}(\sigma)}{\sqrt{C_{\sigma}}}\right| \leq \frac{A}{\sqrt{C_{\sigma_{0}}}}\right) \\
& \leq \iint_{\sqrt{x^{2}+y^{2}} \leq A / \sqrt{C_{\sigma_{0}}}} \frac{1}{2 \pi} e^{-\left(x^{2}+y^{2}\right) / 2} d x d y \\
& \rightarrow 0 \quad \text { as } \sigma_{0} \searrow \frac{1}{2},
\end{aligned}
$$

or, equivalently,

$$
\lim _{\sigma \searrow 1 / 2} \mathbf{P}(|\tilde{X}(\sigma)|>A)=1, \quad \forall A>0 .
$$

This can be interpreted as the weak law of large numbers

$$
\lim _{\sigma \searrow 1 / 2} \tilde{X}(\sigma)=\infty \text { (= point at infinity) in probability }
$$

Our problem is to show the strong law of large numbers or, generally, to view the behavior of $\widetilde{X}(\sigma)$ as $\sigma \searrow \frac{1}{2} \mathbf{P}$-almost surely. Unfortunately, this is still open. 


\section{Path space $C\left(\left(\frac{1}{2}, \infty\right) \rightarrow \mathbb{C}\right)$}

In what follows, restricting the domain of $\widetilde{X}$ to $\left(\frac{1}{2}, \infty\right)$, let us call $\{\widetilde{X}(\sigma)\}_{\sigma>1 / 2}$ the Bohr-Jessen process. Thus, for each $\left(x_{f}\right)_{f \in \mathbb{B}} \in \mathbb{R}^{\mathbb{B}}, \widetilde{X}\left(\cdot,\left(x_{f}\right)_{f \in \mathbb{B}}\right) \in C\left(\left(\frac{1}{2}, \infty\right) \rightarrow\right.$ $\mathbb{C})$, where $C\left(\left(\frac{1}{2}, \infty\right) \rightarrow \mathbb{C}\right)$ is the set of all continuous paths $w:\left(\frac{1}{2}, \infty\right) \ni t \mapsto$ $w(t) \in \mathbb{C}$.

\section{CLAIM 3.1}

We have that $C\left(\left(\frac{1}{2}, \infty\right) \rightarrow \mathbb{C}\right)$ is a Polish space.

\section{Proof}

We divide the proof into four steps:

(1) Let $\mathcal{K}$ be the set of all nonempty compact sets of $\left(\frac{1}{2}, \infty\right)$. For $w \in C\left(\left(\frac{1}{2}, \infty\right) \rightarrow\right.$ C), put

$$
\begin{aligned}
U_{w, K, \varepsilon} & :=\left\{z \in C\left(\left(\frac{1}{2}, \infty\right) \rightarrow \mathbb{C}\right) ; \max _{t \in K}|z(t)-w(t)|<\varepsilon\right\}, \quad K \in \mathcal{K}, \varepsilon>0 \\
\mathcal{B}^{\prime}(w) & :=\left\{U_{w, K, \varepsilon} ; K \in \mathcal{K}, \varepsilon>0\right\}, \\
\mathcal{B}(w) & :=\left\{\begin{array}{ll}
U_{w, K_{1}, \varepsilon_{1}} \cap \cdots \cap U_{w, K_{n}, \varepsilon_{n}} ; & \varepsilon_{1}, \ldots, K_{n}, \ldots \mathcal{K}, \\
n=1,2, \ldots
\end{array}\right\} .
\end{aligned}
$$

Then $\left\{\mathcal{B}^{\prime}(w)\right\}_{w \in C((1 / 2, \infty) \rightarrow \mathbb{C})}$ satisfies the conditions of a subbase for $C\left(\left(\frac{1}{2}\right.\right.$, $\infty) \rightarrow \mathbb{C})$ :

- $w \in U_{f, K, \varepsilon}$,

- $z \in U_{w, K, \varepsilon} \Rightarrow \exists K_{z} \in \mathcal{K}, \exists \varepsilon_{z}>0$ s.t. $U_{z, K_{z}, \varepsilon_{z}} \subset U_{w, K, \varepsilon}$,

so that $\{\mathcal{B}(w)\}_{w \in C((1 / 2, \infty) \rightarrow \mathbb{C})}$ generates a topology $\mathcal{O}$ of $C\left(\left(\frac{1}{2}, \infty\right) \rightarrow \mathbb{C}\right)$ where

$$
\mathcal{O}=\left\{\bigcup_{U \in \mathcal{B}} U ; \mathcal{B} \subset \underset{w \in C((1 / 2, \infty) \rightarrow \mathbb{C})}{\bigcup} \mathcal{B}(w)\right\}
$$

In this case $\{\mathcal{B}(w)\}_{w \in C((1 / 2, \infty) \rightarrow \mathbb{C})}$ becomes a system of local bases of a topological space $\left(C\left(\left(\frac{1}{2}, \infty\right) \rightarrow \mathbb{C}\right), \mathcal{O}\right)$.

(2) $\left(C\left(\left(\frac{1}{2}, \infty\right) \rightarrow \mathbb{C}\right), \mathcal{O}\right)$ is metrizable.

$\odot$ Indeed, taking $\left\{K_{n}\right\}_{n=1}^{\infty} \subset \mathcal{K}$ so that

$$
\begin{aligned}
& K_{n} \subset \stackrel{\circ}{K}_{n+1} \quad(\forall n \geq 1), \\
& \bigcup_{n=1}^{\infty} K_{n}=\left(\frac{1}{2}, \infty\right),
\end{aligned}
$$

we define

$$
d(w, z):=\sum_{n=1}^{\infty} \frac{1}{2^{n}}\left(\max _{t \in K_{n}}|w(t)-z(t)|\right) \wedge 1, \quad z, w \in C\left(\left(\frac{1}{2}, \infty\right) \rightarrow \mathbb{C}\right) .
$$


Then $d(\cdot, *)$ is a metric on $C\left(\left(\frac{1}{2}, \infty\right) \rightarrow \mathbb{C}\right)$, and the topology $\mathcal{O}(d)$ introduced by $d$ coincides with $\mathcal{O}$. The first assertion is obvious. The second assertion is checked in the following way:

Let $\left.w \in C\left(\frac{1}{2}, \infty\right) \rightarrow \mathbb{C}\right)$, and let $\varepsilon>0$. Take $n_{0} \in \mathbb{N}$ so that $1 / 2^{n_{0}}<\varepsilon / 2$. From the implications

$$
\begin{aligned}
z \in U_{w, K_{n_{0}}, \varepsilon / 2 \wedge 1} \Rightarrow \max _{t \in K_{n_{0}}}|z(t)-w(t)|<\frac{\varepsilon}{2} \wedge 1 \\
\Rightarrow d(z, w)=\sum_{n=1}^{\infty} \frac{1}{2^{n}}\left(\max _{t \in K_{n}}|z(t)-w(t)|\right) \wedge 1 \\
\left.=\sum_{n=1}^{n_{0}} \frac{1}{2^{n}} \max _{t \in K_{n}}|z(t)-w(t)|\right) \wedge 1 \\
+\sum_{n=n_{0}+1}^{\infty} \frac{1}{2^{n}}\left(\max _{t \in K_{n}}|z(t)-w(t)|\right) \wedge 1 \\
\leq \sum_{n=1}^{n_{0}} \frac{1}{2^{n}}\left(\max _{t \in K_{n_{0}}}|w(t)-z(t)|\right) \wedge 1 \\
+\sum_{n=n_{0}+1}^{\infty} \frac{1}{2^{n}}\left[\odot K_{n} \subset K_{n_{0}}\left(1 \leq n \leq n_{0}\right)\right] \\
\leq\left(\sum_{n=1}^{n_{0}} \frac{1}{2^{n}}\right) \max _{t \in K_{n_{0}}}|z(t)-w(t)|+\frac{1}{2^{n_{0}}} \\
<\frac{\varepsilon}{2}+\frac{\varepsilon}{2}=\varepsilon,
\end{aligned}
$$

it follows that $U_{w, K_{n_{0}}, \varepsilon / 2 \wedge 1} \subset N_{w, \varepsilon}:=\{z ; d(z, w)<\varepsilon\}$, which implies that $\mathcal{O}(d) \subset \mathcal{O}$.

Next we let $w \in C\left(\left(\frac{1}{2}, \infty\right) \rightarrow \mathbb{C}\right)$, we let $K \in \mathcal{K}$, and we let $\varepsilon>0$. Since $K \subset$ $\left(\frac{1}{2}, \infty\right)=\bigcup_{n=1}^{\infty} \stackrel{\circ}{K}{ }_{n}$ and $K$ is compact, $\exists n_{0} \in \mathbb{N}$ such that $K \subset \stackrel{\circ}{K_{n_{0}}} \subset K_{n_{0}}$. From the implications

$$
\begin{aligned}
z \in N_{w,(\varepsilon \wedge 1) / 2^{n_{0}}} \Rightarrow & d(z, w)<\frac{\varepsilon \wedge 1}{2^{n_{0}}} \\
& \sum_{n=1}^{\infty} \frac{1}{2^{n}}\left(\max _{t \in K_{n}}|z(t)-w(t)|\right) \wedge 1 \\
\Rightarrow & \frac{1}{2^{n_{0}}}\left(\max _{t \in K_{n_{0}}}|z(t)-w(t)|\right) \wedge 1<\frac{\varepsilon \wedge 1}{2^{n_{0}}} \\
\Rightarrow & \left.\max _{t \in K_{n_{0}}}|z(t)-w(t)|\right) \wedge 1<\varepsilon \wedge 1 \leq 1 \\
\Rightarrow & \max _{t \in K_{n_{0}}}|z(t)-w(t)|<\varepsilon \wedge 1 \leq \varepsilon
\end{aligned}
$$




$$
\begin{aligned}
& \Rightarrow \max _{t \in K}|z(t)-w(t)|<\varepsilon \\
& \Rightarrow z \in U_{w, K, \varepsilon}
\end{aligned}
$$

it follows that $N_{w,(\varepsilon \wedge 1) / 2^{n_{0}}} \subset U_{w, K, \varepsilon}$, which implies that $\mathcal{O} \subset \mathcal{O}(d)$. Therefore, we have $\mathcal{O}(d)=\mathcal{O}$.

(3) $\left(C\left(\left(\frac{1}{2}, \infty\right) \rightarrow \mathbb{C}\right), d\right)$ is complete.

$\odot$ Let $\left\{w_{k}\right\}_{k=1}^{\infty} \subset C\left(\left(\frac{1}{2}, \infty\right) \rightarrow \mathbb{C}\right)$ be a $d$-Cauchy sequence, that is, $\lim _{k, l \rightarrow \infty} d\left(w_{k}, w_{l}\right)=0$. By $(3.3)$,

$$
\lim _{k, l \rightarrow \infty} \max _{t \in K_{n}}\left|w_{k}(t)-w_{l}(t)\right|=0 \quad(\forall n \in \mathbb{N}) .
$$

This tells us that $\left\{w_{k}(t)\right\}_{k=1}^{\infty}$ is a Cauchy sequence of $\mathbb{C}$ for each $t \in\left(\frac{1}{2}, \infty\right)$. Thus, $\exists w(t) \in \mathbb{C}$ such that $\lim _{k \rightarrow \infty} w_{k}(t)=w(t)$. Since, for $n \in \mathbb{N}$ and $t \in K_{n}$,

$$
2^{n} d\left(w_{k}, w_{l}\right) \geq\left|w_{k}(t)-w_{l}(t)\right| \wedge 1,
$$

letting $l \rightarrow \infty$ yields that

$$
\left|w_{k}(t)-w(t)\right| \wedge 1 \leq 2^{n} \lim _{l \rightarrow \infty} d\left(w_{k}, w_{l}\right) \quad\left(t \in K_{n}\right) .
$$

Taking the sup in $t \in K_{n}$ yields that

$$
\left(\sup _{t \in K_{n}}\left|w_{k}(t)-w(t)\right|\right) \wedge 1 \leq 2^{n} \lim _{l \rightarrow \infty} d\left(w_{k}, w_{l}\right) .
$$

Letting $k \rightarrow \infty$, we see that

$$
\lim _{k \rightarrow \infty} \sup _{t \in K_{n}}\left|w_{k}(t)-w(t)\right|=0 \quad(\forall n \in \mathbb{N}) .
$$

This implies that $w \in C\left(\left(\frac{1}{2}, \infty\right) \rightarrow \mathbb{C}\right)$, so that we have $\lim _{k \rightarrow \infty} d\left(w_{k}, w\right)=0$, which shows the completeness of $\left(C\left(\left(\frac{1}{2}, \infty\right) \rightarrow \mathbb{C}\right), d\right)$.

(4) $\left(C\left(\left(\frac{1}{2}, \infty\right) \rightarrow \mathbb{C}\right), d\right)$ is separable.

$\odot$ Let $\mathbb{C}[t]$ be the set of all polynomials of variable $t$ with complex coefficients. From Weierstrass's approximation theorem, it follows that, $\forall w \in C\left(\left(\frac{1}{2}, \infty\right) \rightarrow \mathbb{C}\right)$, $\forall K \in \mathcal{K}$, and $\forall \varepsilon>0$,

$$
\exists p \in \mathbb{C}[t] \quad \text { s.t. } \max _{t \in K}|f(t)-p(t)|<\varepsilon .
$$

Then let

$$
\begin{aligned}
(\mathbb{Q} & +\sqrt{-1} \mathbb{Q})[t] \\
& :=\left\{p \in \mathbb{C}[t] ; \begin{array}{l}
\text { all the coefficients of } p \text { are rational, i.e., of the form } \\
r+\sqrt{-1} s(r, s \in \mathbb{Q})
\end{array}\right\} .
\end{aligned}
$$

Clearly $(\mathbb{Q}+\sqrt{-1} \mathbb{Q})[t]$ is a countable set, and from the above $\left\{\left.p\right|_{(1 / 2, \infty)} ; p \in\right.$ $(\mathbb{Q}+\sqrt{-1} \mathbb{Q})[t]\}$ is dense in $C\left(\left(\frac{1}{2}, \infty\right) \rightarrow \mathbb{C}\right)$. Therefore, $\left(C\left(\left(\frac{1}{2}, \infty\right) \rightarrow \mathbb{C}\right), d\right)$ is separable. 
We have that $\tilde{X}$ is a $C\left(\left(\frac{1}{2}, \infty\right) \rightarrow \mathbb{C}\right)$-valued random variable; that is, the mapping $\mathbb{R}^{\mathbb{B}} \ni\left(x_{f}\right)_{f \in \mathbb{B}} \mapsto \tilde{X}\left(\cdot,\left(x_{f}\right)_{f \in \mathbb{B}}\right) \in C\left(\left(\frac{1}{2}, \infty\right) \rightarrow \mathbb{C}\right)$ is $\mathcal{B}_{K}\left(\mathbb{R}^{\mathbb{B}}\right)$-measurable. The image measure of $\mathbf{P}$ by this mapping is denoted by $\mathbf{P} \circ \widetilde{X}^{-1}$ as usual.

\section{CLAIM 3.2}

Let $M_{n}(n=1,2, \ldots)$ and $M$ be probability measures on $C\left(\left(\frac{1}{2}, \infty\right) \rightarrow \mathbb{C}\right)$. If, for $\frac{1}{2}<\sigma_{1}<\sigma_{2}<\infty$ and $\forall \Phi: C\left(\left[\sigma_{1}, \sigma_{2}\right] \rightarrow \mathbb{C}\right) \rightarrow \mathbb{R}$ that are bounded uniformly continuous,

$$
\begin{aligned}
& \int_{C((1 / 2, \infty) \rightarrow \mathbb{C})} \Phi\left(\left.w\right|_{\left[\sigma_{1}, \sigma_{2}\right]}\right) M_{n}(d w) \\
& \quad \rightarrow \int_{C((1 / 2, \infty) \rightarrow \mathbb{C})} \Phi\left(\left.w\right|_{\left[\sigma_{1}, \sigma_{2}\right]}\right) M(d w) \text { as } n \rightarrow \infty,
\end{aligned}
$$

then $M_{n}$ is weakly convergent to $M$ as $n \rightarrow \infty$. Here $\left.w\right|_{\left[\sigma_{1}, \sigma_{2}\right]}$ is the restriction of $w$ to $\left[\sigma_{1}, \sigma_{2}\right]$.

Proof

Take $\left\{K_{n}\right\}_{n=1}^{\infty} \subset \mathcal{K}$ as

$$
K_{n}=\left[\frac{1}{2}+\frac{1}{n+1}, n+1\right], \quad n \in \mathbb{N} .
$$

Clearly (3.1) and (3.2) are satisfied, so by (3.3) we define a metric $d(\cdot, *)$ on $C\left(\left(\frac{1}{2}, \infty\right) \rightarrow \mathbb{C}\right)$. For each $m \in \mathbb{N}$ and $w \in C\left(K_{m} \rightarrow \mathbb{C}\right)$, let $w_{m} \in C\left(\left(\frac{1}{2}, \infty\right) \rightarrow \mathbb{C}\right)$ be

$$
w_{m}(t):=w\left(\left(t \vee\left(\frac{1}{2}+\frac{1}{m+1}\right)\right) \wedge(m+1)\right) .
$$

Since, for $w, z \in C\left(K_{m} \rightarrow \mathbb{C}\right)$,

$$
\max _{t \in K_{n}}\left|w_{m}(t)-z_{m}(t)\right| \begin{cases}\leq \max _{t \in K_{m}}|w(t)-z(t)| & \text { if } n<m, \\ =\max _{t \in K_{m}}|w(t)-z(t)| & \text { if } n \geq m,\end{cases}
$$

it follows that

$$
d\left(w_{m}, z_{m}\right) \leq \max _{t \in K_{m}}|w(t)-z(t)| .
$$

Also, since, for $w \in C\left(\left(\frac{1}{2}, \infty\right) \rightarrow \mathbb{C}\right)$,

$$
w(t)=\left(\left.w\right|_{K_{m}}\right)_{m}(t) \quad\left(t \in K_{n}, n \leq m\right),
$$

it follows that

$$
d\left(w,\left(\left.w\right|_{K_{m}}\right)_{m}\right) \leq \frac{1}{2^{m}} .
$$

Now let $M_{n}(n=1,2, \ldots)$ and $M$ be those probability measures on $C\left(\left(\frac{1}{2}, \infty\right) \rightarrow \mathbb{C}\right)$ as in Claim 3.2. Let $\Phi: C\left(\left(\frac{1}{2}, \infty\right) \rightarrow \mathbb{C}\right) \rightarrow \mathbb{R}$ be bounded uniformly continuous. For each $m \in \mathbb{N}$, define $\Phi_{m}: C\left(K_{m} \rightarrow \mathbb{C}\right) \rightarrow \mathbb{R}$ by $\Phi_{m}(w):=$ $\Phi\left(w_{m}\right)$. By (3.4), $\Phi_{m}$ is also bounded uniformly continuous. Also, by (3.5),

$$
\left|\Phi(w)-\Phi\left(\left(\left.w\right|_{K_{m}}\right)_{m}\right)\right| \leq \sup \left\{\left|\Phi(z)-\Phi\left(z^{\prime}\right)\right| ; d\left(z, z^{\prime}\right) \leq \frac{1}{2^{m}}\right\} .
$$


Thus,

$$
\begin{aligned}
& \left|\int_{C((1 / 2, \infty) \rightarrow \mathbb{C})} \Phi(w) M_{n}(d w)-\int_{C((1 / 2, \infty) \rightarrow \mathbb{C})} \Phi(w) M(d w)\right| \\
& =\mid \int_{C((1 / 2, \infty) \rightarrow \mathbb{C})}\left(\Phi(w)-\Phi\left(\left(\left.w\right|_{K_{m}}\right)_{m}\right)\right) M_{n}(d w) \\
& \quad+\int_{C((1 / 2, \infty) \rightarrow \mathbb{C})} \Phi_{m}\left(\left.w\right|_{K_{m}}\right) M_{n}(d w)-\int_{C((1 / 2, \infty) \rightarrow \mathbb{C})} \Phi_{m}\left(\left.w\right|_{K_{m}}\right) M(d w) \\
& \quad+\int_{C((1 / 2, \infty) \rightarrow \mathbb{C})}\left(\Phi\left(\left(\left.w\right|_{K_{m}}\right)_{m}\right)-\Phi(w)\right) M(d w) \mid \\
& \leq 2 \sup \left\{\left|\Phi(z)-\Phi\left(z^{\prime}\right)\right| ; d\left(z, z^{\prime}\right) \leq \frac{1}{2^{m}}\right\} \\
& \quad+\left|\int_{C((1 / 2, \infty) \rightarrow \mathbb{C})} \Phi_{m}\left(\left.w\right|_{K_{m}}\right) M_{n}(d w)-\int_{C((1 / 2, \infty) \rightarrow \mathbb{C})} \Phi_{m}\left(\left.w\right|_{K_{m}}\right) M(d w)\right| \\
& \begin{aligned}
\text { first } n \rightarrow \infty \\
\text { second } m \rightarrow \infty
\end{aligned}
\end{aligned}
$$

which shows the assertion of Claim 3.2.

\section{Bohr-Jessen functional limit theorem}

At the moment, we do not know about the zeros of $\zeta(\cdot)$ completely. To define the log zeta function, we thus need some explanations. Put

$$
\Gamma:=\{t \in \mathbb{R} \backslash\{0\} ; \exists \sigma \in(0,1) \text { s.t. } \zeta(\sigma+\sqrt{-1} t)=0\} .
$$

At most, $\Gamma$ is countable, since each point of $\Gamma$ is isolated, and $\Gamma$ is symmetric, that is, $-\Gamma=\Gamma$. For each $t \in \Gamma,\{\sigma \in(0,1) ; \zeta(\sigma+\sqrt{-1} t)=0\}$ is symmetric relative to $\sigma=\frac{1}{2} ;$ in other words,

$$
\zeta(\sigma+\sqrt{-1} t)=0 \quad \Longleftrightarrow \quad \zeta(1-\sigma+\sqrt{-1} t)=0 .
$$

\section{DEFINITION 4.1}

For each $t \in \Gamma$, put

$$
\sigma_{t}:=\sup \{\sigma \in(0,1) ; \zeta(\sigma+\sqrt{-1} t)=0\} .
$$

From the above, note that $\frac{1}{2} \leq \sigma_{t}<1$. We then define

$$
G:=\mathbb{C} \backslash\left(\bigcup_{t \in \Gamma}\left\{\sigma+\sqrt{-1} t ;-\infty<\sigma \leq \sigma_{t}\right\} \cup(-\infty, 1]\right) .
$$

\section{DEFINITION 4.2}

We have that $G$ is a simply connected domain, $G \supset\{s \in \mathbb{C} ; \operatorname{Re} s>1\}$, and $\zeta(s) \neq 0$ $(s \in G)$. Then

$$
l(s)=\log \frac{\pi^{2}}{6}+\int_{2}^{s} \frac{\zeta^{\prime}(z)}{\zeta(z)} d z, \quad s \in G,
$$


is holomorphic and satisfies that $l(2)=\log \left(\pi^{2} / 6\right)$ and $\zeta(s)=e^{l(s)}(s \in G)$. This $l(\cdot)$ is called the $\log$ zeta function and is denoted by $\log \zeta(\cdot)$.

Recall that $U_{T}$ was a real-valued random variable uniformly distributed on $[-T, T]$. Thus,

$$
P\left(U_{T} \in E\right)=P_{T}(E), \quad E \in \mathcal{B}(\mathbb{R})(\text { see }(2.1)),
$$

where $(\Omega, \mathcal{F}, P)$ is a probability space on which $U_{T}$ is defined. For $\frac{1}{2}<\sigma<\infty$, we defined $\widetilde{X}_{T}(\sigma)$ by (1.3). From the implications

$$
\begin{aligned}
U_{T} \notin \Gamma \cup\{0\} & \Rightarrow \sigma+\sqrt{-1} U_{T} \in G(\forall \sigma \in \mathbb{R}) \\
& \Rightarrow \widetilde{X}_{T}(\cdot) \text { is continuous on }\left(\frac{1}{2}, \infty\right)
\end{aligned}
$$

and $U_{T} \notin \Gamma \cup\{0\}$ almost surely, it is seen that $\tilde{X}_{T}(\cdot) \in C\left(\left(\frac{1}{2}, \infty\right) \rightarrow \mathbb{C}\right)$ almost surely.

\section{THEOREM 4.1}

The distribution of $\left\{\widetilde{X}_{T}(\sigma)\right\}_{\sigma>1 / 2}$ is weakly convergent to that of $\{\widetilde{X}(\sigma)\}_{\sigma>1 / 2}$ as $T \rightarrow \infty$. In other words, $P \circ \widetilde{X}_{T}^{-1} \rightarrow \mathbf{P} \circ \widetilde{X}^{-1}$ as $T \rightarrow \infty$.

Since, for each $\sigma \in\left(\frac{1}{2}, \infty\right)$, we called the convergence

$$
\widetilde{X}_{T}(\sigma) \rightarrow \widetilde{X}(\sigma) \text { in law as } T \rightarrow \infty
$$

the Bohr-Jessen limit theorem, let us call the above theorem the Bohr-Jessen functional limit theorem, from which the name Bohr-Jessen process of $\{\widetilde{X}(\sigma)\}_{\sigma>1 / 2}$ comes.

For the proof of Theorem 4.1, we need a definition which gives an approximation of $\zeta(\cdot)$.

\section{DEFINITION 4.3}

For $N \in \mathbb{N}$, put

$$
\zeta_{N}(s):=\prod_{i=1}^{N} \frac{1}{1-1 / p_{i}^{s}}, \quad \operatorname{Re} s>0(\text { see }(1.2)) .
$$

Clearly, $\zeta_{N}(\cdot)$ is holomorphic and has no zeros on $\{s \in \mathbb{C} ; \operatorname{Re} s>0\}$. And

$$
\log \zeta_{N}(s)=\sum_{i=1}^{N}-\log \left(1-\frac{1}{p_{i}^{s}}\right)
$$

holds (see [18, Definition 5.3]). ${ }^{\dagger}$

\footnotetext{
${ }^{\dagger} \log \zeta_{N}(\cdot)$ is defined similarly to $\log \zeta(\cdot)$; i.e., $\log \zeta_{N}(s)=\log \zeta_{N}(2)+\int_{2}^{s}\left(\zeta_{N}^{\prime}(z) / \zeta_{N}(z)\right) d z$, where $\log \zeta_{N}(2)$ is a usual logarithm.
} 
For $T>0$ and $N \in \mathbb{N}$, we define a continuous process $\left\{\widetilde{X}_{T, N}(\sigma)\right\}_{\sigma>1 / 2}$ by

$$
\widetilde{X}_{T, N}(\sigma):=\log \zeta_{N}\left(\sigma+\sqrt{-1} U_{T}\right)
$$

We divide the proof of Theorem 4.1 into four steps.

STEP 1

For $\frac{1}{2}<\sigma_{1}<\sigma_{2}<\infty$ and $\forall \varepsilon>0$,

$$
\lim _{N \rightarrow \infty} \limsup _{T \rightarrow \infty} P\left(\sup _{\sigma_{1} \leq \sigma \leq \sigma_{2}}\left|\widetilde{X}_{T}(\sigma)-\widetilde{X}_{T, N}(\sigma)\right| \geq \varepsilon\right)=0 .
$$

For the proof, we need the following proposition.

PROPOSITION 4.1 (CARLSON'S MEAN VALUE THEOREM)

Let $\sum_{n=1}^{\infty} a_{n} / n^{s}$ be a Dirichlet series absolutely convergent on $\{s \in \mathbb{C} ; \operatorname{Re} s>1\}$. Suppose that it is analytically continuable to a meromorphic function $f(\cdot)$ on $\{s \in \mathbb{C} ; \operatorname{Re} s \geq \alpha\}$ (where $-\infty<\alpha<1$ ), suppose that $f(\cdot)$ is holomorphic except at $s=1$ which is a removable singularity or a pole of $f(\cdot)$, and suppose that $f(s)=O\left((|\operatorname{Im} s|+2)^{C}\right)$ except in some neighborhood of $s=1$ where $C$ is a positive constant. If

$$
\int_{-T}^{T}|f(\alpha+\sqrt{-1} t)|^{2} d t=O(T) \quad \text { as } T \rightarrow \infty
$$

the following hold.

(i) For $\alpha<\sigma<\infty, \sum_{n=1}^{\infty}\left|a_{n}\right|^{2} / n^{2 \sigma}<\infty$.

(ii) For $\alpha<\sigma_{1}<\sigma_{2}<\infty$,

$$
\left.\sup _{\sigma_{1} \leq \sigma \leq \sigma_{2}}\left|\frac{1}{T} \int_{2}^{T}\right| f(\sigma+\sqrt{-1} t)\right|^{2} d t-\sum_{n=1}^{\infty} \frac{\left|a_{n}\right|^{2}}{n^{2 \sigma}} \mid \rightarrow 0 \quad \text { as } T \rightarrow \infty \text {. }
$$

For the proof, see [14, Theorem 9.6] or [18, Theorem 6.3].

Proof of Step 1

We divide the proof into seven steps.

(1) Fix $N \in \mathbb{N}$. For simplicity, put

$$
f_{N}(s):=\frac{\zeta}{\zeta_{N}}(s)-1, \quad \operatorname{Re} s>0,
$$

where $f_{N}$ is meromorphic on $\{s \in \mathbb{C} ; \operatorname{Re} s>0\}$ and is holomorphic except at $s=1$ which is a simple pole of $f_{N}$. By (1.2),

$$
f_{N}(s)=\sum_{\substack{n \geq 2 ; \\ p_{1} \nmid n, \ldots, p_{N} \nmid n}} \frac{1}{n^{s}}, \quad \operatorname{Re} s>1 .
$$

This tells us that $f_{N}(\cdot)$ is expanded in a Dirichlet series on $\{s \in \mathbb{C} ; \operatorname{Re} s>1\}$ and its convergence is absolute. 
Clearly, for $\operatorname{Re} s \geq \frac{1}{2}, s \neq 1$,

$$
\begin{aligned}
\left|f_{N}(s)\right| & =\left|\zeta(s) \prod_{i=1}^{N}\left(1-\frac{1}{p_{i}^{s}}\right)-1\right| \leq|\zeta(s)| \prod_{i=1}^{N}\left(1+\frac{1}{p_{i}^{\operatorname{Re} s}}\right)+1 \\
& \leq|\zeta(s)| \prod_{i=1}^{N}\left(1+\frac{1}{p_{i}^{1 / 2}}\right)+1 .
\end{aligned}
$$

Here we note that

(a) $\sup \left\{\frac{|\zeta(s)|}{(2+|\operatorname{Im} s|)^{3}} ; \operatorname{Re} s \geq-\frac{3}{2},|s-1| \geq \frac{1}{3}\right\}<\infty$ (see [18, Lemma 6.2]),

(b) for $\frac{1}{2}<\alpha<1, \int_{1 \leq|t| \leq T}|\zeta(\alpha+\sqrt{-1} t)|^{2} d t \sim 2 T \zeta(2 \alpha)$ as $T \rightarrow \infty$ (see [18, Claim 6.3]).

From these and (4.8), it is easily seen that

$$
\begin{gathered}
\sup \left\{\frac{\left|f_{N}(s)\right|}{(2+|\operatorname{Im} s|)^{3}} ; \operatorname{Re} s \geq \frac{1}{2},|s-1| \geq \frac{1}{3}\right\}<\infty, \\
\int_{-T}^{T}\left|f_{N}(\alpha+\sqrt{-1} t)\right|^{2} d t=O(T) \quad \text { as } T \rightarrow \infty .
\end{gathered}
$$

Therefore, it turns out that $f_{N}(\cdot)$ satisfies the assumptions in Proposition 4.1 above. We thus apply this proposition to have that, for $\frac{1}{2}<\alpha<1$ and $\alpha<\sigma_{1}<$ $\sigma_{2}<\infty$

$$
\left.\lim _{T \rightarrow \infty} \sup _{\sigma_{1} \leq \sigma \leq \sigma_{2}}\left|\frac{1}{T} \int_{2}^{T}\right| f_{N}(\sigma+\sqrt{-1} t)\right|^{2} d t-\sum_{\substack{n \geq 2 ; \\ p_{1} \nmid n, \ldots, p_{N} \nmid n}} \frac{1}{n^{2 \sigma}} \mid=0 .
$$

(2) In the following, fix $\frac{1}{2}<\sigma_{1}<\frac{3}{4}<\frac{3}{2}<\sigma_{2}<\infty$. Let $\sigma_{0}:=\frac{1}{2}\left(\frac{1}{2}+\sigma_{1}\right)$, and let $\sigma_{3}:=\sigma_{2}+\frac{1}{2}$. Clearly, $\frac{1}{2}<\sigma_{0}<\sigma_{1}<\frac{3}{4}<\frac{3}{2}<\sigma_{2}<\sigma_{3}$. By (1),

$$
\left.\lim _{T \rightarrow \infty} \sup _{\sigma_{0} \leq \sigma \leq \sigma_{3}}\left|\frac{1}{T} \int_{\frac{1}{2}}^{T}\right| f_{N}(\sigma+\sqrt{-1} t)\right|^{2} d t-\sum_{\substack{n \geq 2 ; \\ p_{1} \nmid n, \ldots, p_{N} \nmid n}} \frac{1}{n^{2 \sigma}} \mid=0 .
$$

Fix $0<\eta<1 \wedge\left(\left(\sigma_{1}-\frac{1}{2}\right) / 2\left[\left(\sigma_{3}-\sigma_{0}\right) / \pi\right]^{1 / 2}\right)^{5}=\left(\left(\sigma_{1}-\frac{1}{2}\right) / 2\left[\left(\sigma_{3}-\sigma_{0}\right) /\right.\right.$ $\left.\pi]^{1 / 2}\right)^{5}$. Take $N_{0}=N_{0}\left(\sigma_{0}, \eta\right) \in \mathbb{N}$ so that

$$
\sum_{n>p_{N_{0}}} \frac{1}{n^{2 \sigma_{0}}}<\frac{\eta}{2}
$$

Since $n>p_{N}$ provided $n \geq 2$ and $p_{1} \nmid n, \ldots, p_{N} \nmid n$,

$$
\sum_{\substack{n \geq 2 ; \\ p_{1} \nmid n, \ldots, p_{N} \nmid n}} \frac{1}{n^{2 \sigma}} \leq \sum_{n>p_{N}} \frac{1}{n^{2 \sigma}} \quad\left(\sigma>\frac{1}{2}\right) .
$$

From the convergence above, it follows that, $\forall N \geq N_{0}$, 


$$
\begin{aligned}
& \exists T_{0}\left(N, \sigma_{1}, \sigma_{2}, \eta\right) \in[2, \infty) \\
& \text { s.t. }\left.\sup _{\sigma_{0} \leq \sigma \leq \sigma_{3}}\left|\frac{1}{T} \int_{1 / 2}^{T}\right| f_{N}(\sigma+\sqrt{-1} t)\right|^{2} d t-\sum_{\substack{n \geq 2 ; \\
p_{1} \nmid n, \ldots, p_{N} \nmid n}} \frac{1}{n^{2 \sigma}} \mid<\frac{\eta}{2}, \\
& \forall T \geq T_{0}\left(N, \sigma_{1}, \sigma_{2}, \eta\right) .
\end{aligned}
$$

In conjunction with (4.9), we see that, $\forall T \geq T_{0}\left(N, \sigma_{1}, \sigma_{2}, \eta\right)$,

$$
\begin{aligned}
\sup _{\sigma_{0} \leq \sigma \leq \sigma_{3}} & \frac{1}{T} \int_{1 / 2}^{T}\left|f_{N}(\sigma+\sqrt{-1} t)\right|^{2} d t \\
& =\sup _{\sigma_{0} \leq \sigma \leq \sigma_{3}}\left(\frac{1}{T} \int_{1 / 2}^{T}\left|f_{N}(\sigma+\sqrt{-1} t)\right|^{2} d t-\sum_{\substack{n \geq 2 ; \\
p_{1} \nmid n, \ldots, p_{N} \nmid n}} \frac{1}{n^{2 \sigma}}+\sum_{\substack{n \geq 2 ; \\
p_{1} \nmid n, \ldots, p_{N} \nmid n}} \frac{1}{n^{2 \sigma}}\right) \\
& <\frac{\eta}{2}+\frac{\eta}{2}=\eta .
\end{aligned}
$$

(3) Let $N \geq N_{0}\left(\sigma_{0}, \eta\right)$, and let $T \geq T_{0}\left(N, \sigma_{1}, \sigma_{2}, \eta\right)$. Put $K_{T} \subset\{1, \ldots,\lfloor T\rfloor-1\}$ as

$$
K_{T}:=\left\{k \in\{1, \ldots,\lfloor T\rfloor-1\} ; \int_{k-1 / 2}^{k+1 / 2} d t \int_{\sigma_{0}}^{\sigma_{3}}\left|f_{N}(\sigma+\sqrt{-1} t)\right|^{2} d \sigma\right\} .
$$

Let $\delta=\left[\left(\sigma_{3}-\sigma_{0}\right) / \pi\right]^{1 / 2} \eta^{1 / 5}$ [note that $\left.0<\delta<\frac{1}{2}\left(\sigma_{1}-\frac{1}{2}\right)\right]$, and put $J_{T} \subset\left[\frac{1}{2}+\right.$ $\left.\delta, T-\frac{1}{2}-\delta\right]$ as

$$
J_{T}:=\sum_{k \in K_{T}}\left[k-\frac{1}{2}+\delta, k+\frac{1}{2}-\delta\right] .
$$

Then $\mu\left(J_{T}\right) \geq(\lfloor T\rfloor-1-\sqrt{\eta} T)(1-2 \delta)$. For, by $(2)$,

$$
\begin{aligned}
\left(\sigma_{3}-\sigma_{0}\right) \eta T & \geq \int_{\sigma_{0}}^{\sigma_{3}} d \sigma \int_{1 / 2}^{T}\left|f_{N}(\sigma+\sqrt{-1} t)\right|^{2} d t \\
& =\int_{1 / 2}^{T} d t \int_{\sigma_{0}}^{\sigma_{3}}\left|f_{N}(\sigma+\sqrt{-1} t)\right|^{2} d \sigma \\
& \geq \int_{1 / 2}^{\lfloor T\rfloor-1 / 2} d t \int_{\sigma_{0}}^{\sigma_{3}}\left|f_{N}(\sigma+\sqrt{-1} t)\right|^{2} d \sigma \\
& =\sum_{k=1}^{\lfloor T\rfloor-1} \int_{k-1 / 2}^{k+1 / 2} d t \int_{\sigma_{0}}^{\sigma_{3}}\left|f_{N}(\sigma+\sqrt{-1} t)\right|^{2} d \sigma \\
& \geq \sum_{k \in\{1, \ldots,\lfloor T\rfloor-1\} \backslash K_{T}} \int_{k-1 / 2}^{k+1 / 2} d t \int_{\sigma_{0}}^{\sigma_{3}}\left|f_{N}(\sigma+\sqrt{-1} t)\right|^{2} d \sigma
\end{aligned}
$$




$$
\begin{aligned}
& \geq \sum_{k \in\{1, \ldots,\lfloor T\rfloor-1\} \backslash K_{T}}\left(\sigma_{3}-\sigma_{0}\right) \sqrt{\eta} \\
& =\#\left(\{1, \ldots,\lfloor T\rfloor-1\} \backslash K_{T}\right)\left(\sigma_{3}-\sigma_{0}\right) \sqrt{\eta} \\
& =\left(\lfloor T\rfloor-1-\# K_{T}\right)\left(\sigma_{3}-\sigma_{0}\right) \sqrt{\eta},
\end{aligned}
$$

which implies that $\# K_{T} \geq\lfloor T\rfloor-1-\sqrt{\eta} T$. Since $\mu\left(J_{T}\right)=\left(\# K_{T}\right)(1-2 \delta)$, the required estimate follows at once.

(4) Let $N$ and $T$ be as above. Then $\left|f_{N}(\sigma+\sqrt{-1} t)\right|<\eta^{1 / 20}, \quad(\sigma, t) \in$ $\left[\sigma_{1}, \sigma_{2}\right] \times J_{T}$.

$\odot$ Fix $(\sigma, t) \in\left[\sigma_{1}, \sigma_{2}\right] \times J_{T}$. By the definition of $J_{T}$,

$$
\exists k \in K_{T} \quad \text { s.t. }(\sigma, t) \in\left[\sigma_{1}, \sigma_{2}\right] \times\left[k-\frac{1}{2}+\delta, k+\frac{1}{2}-\delta\right] .
$$

Then, from the implications

$$
\begin{aligned}
& \left|s^{\prime}-(\sigma+\sqrt{-1} t)\right| \leq \delta \\
& \quad \Rightarrow\left|\operatorname{Re} s^{\prime}-\sigma\right| \leq \delta, \quad\left|\operatorname{Im} s^{\prime}-t\right| \leq \delta \\
& \Rightarrow \sigma-\delta \leq \operatorname{Re} s^{\prime} \leq \sigma+\delta, \quad t-\delta \leq \operatorname{Im} s^{\prime} \leq t+\delta \\
& \quad \Rightarrow \sigma_{1}-\delta \leq \operatorname{Re} s^{\prime} \leq \sigma_{2}+\delta, \quad k-\frac{1}{2} \leq \operatorname{Im} s^{\prime} \leq k+\frac{1}{2} \\
& \quad \Rightarrow \sigma_{0} \leq \operatorname{Re} s^{\prime} \leq \sigma_{3}, \quad k-\frac{1}{2} \leq \operatorname{Im} s^{\prime} \leq k+\frac{1}{2},
\end{aligned}
$$

it follows that

$$
\begin{aligned}
& \overline{\delta \text {-neighborhood of } \sigma+\sqrt{-1} t} \\
& \quad=\left\{s^{\prime} ;\left|s^{\prime}-(\sigma+\sqrt{-1} t)\right| \leq \delta\right\} \\
& \quad \subset\left\{s^{\prime}=\sigma^{\prime}+\sqrt{-1} t^{\prime} ;\left(\sigma^{\prime}, t^{\prime}\right) \in\left[\sigma_{0}, \sigma_{3}\right] \times\left[k-\frac{1}{2}, k+\frac{1}{2}\right]\right\} .
\end{aligned}
$$

By Cauchy's integral representation,

$$
\begin{aligned}
& f_{N}(\sigma+\sqrt{-1} t)^{2} \\
& \quad=\frac{1}{2 \pi \sqrt{-1}} \int_{\left|s^{\prime}-(\sigma+\sqrt{-1} t)\right|=r} \frac{f_{N}\left(s^{\prime}\right)^{2}}{s^{\prime}-(\sigma+\sqrt{-1} t)} d s^{\prime} \\
& \quad=\frac{1}{2 \pi} \int_{0}^{2 \pi} f_{N}\left(\sigma+\sqrt{-1} t+r e^{\sqrt{-1} \theta}\right)^{2} d \theta, \quad 0<r \leq \delta .
\end{aligned}
$$

Taking the absolute value, we have that

$$
\left|f_{N}(\sigma+\sqrt{-1} t)\right|^{2} \leq \frac{1}{2 \pi} \int_{0}^{2 \pi}\left|f_{N}\left(\sigma+\sqrt{-1} t+r e^{\sqrt{-1} \theta}\right)\right|^{2} d \theta .
$$

Multiplying this by $r$ and then integrating it in $r \in[0, \delta]$, we have that 


$$
\begin{aligned}
\mid f_{N} & \left.(\sigma+\sqrt{-1} t)\right|^{2} \frac{\delta^{2}}{2} \\
& =\int_{0}^{\delta}\left|f_{N}(\sigma+\sqrt{-1} t)\right|^{2} r d r \\
& \leq \int_{0}^{\delta} r d r \frac{1}{2 \pi} \int_{0}^{2 \pi}\left|f_{N}\left(\sigma+\sqrt{-1} t+r e^{\sqrt{-1} \theta}\right)\right|^{2} d \theta \\
& =\frac{1}{2 \pi} \int_{0}^{2 \pi} \int_{0}^{\delta}\left|f_{N}\left(\sigma+\sqrt{-1} t+r e^{\sqrt{-1} \theta}\right)\right|^{2} r d r d \theta \\
& =\frac{1}{2 \pi} \iint_{\left|\sigma^{\prime}+\sqrt{-1} t^{\prime}-(\sigma+\sqrt{-1} t)\right| \leq \delta}\left|f_{N}\left(\sigma^{\prime}+\sqrt{-1} t^{\prime}\right)\right|^{2} d \sigma^{\prime} d t^{\prime} \\
& \leq \frac{1}{2 \pi} \int_{k-1 / 2}^{k+1 / 2} d t^{\prime} \int_{\sigma_{0}}^{\sigma_{3}}\left|f_{N}\left(\sigma^{\prime}+\sqrt{-1} t^{\prime}\right)\right|^{2} d \sigma^{\prime} \\
& \leq \frac{1}{2 \pi}\left(\sigma_{3}-\sigma_{0}\right) \sqrt{\eta}\left[\odot \text { since } k \in K_{T}\right] .
\end{aligned}
$$

Thus, we obtain

$$
\begin{aligned}
\left|f_{N}(\sigma+\sqrt{-1} t)\right| & <\left(\frac{2}{\delta^{2}} \cdot \frac{1}{2 \pi}\left(\sigma_{3}-\sigma_{0}\right) \sqrt{\eta}\right)^{1 / 2} \\
& =\left(\frac{\sigma_{3}-\sigma_{0}}{\pi}\right)^{1 / 2} \frac{\eta^{1 / 4}}{\delta} \\
& =\eta^{1 / 20} \quad\left[\odot \delta=\left(\frac{\sigma_{3}-\sigma_{0}}{\pi}\right)^{1 / 2} \eta^{1 / 5}\right] .
\end{aligned}
$$

(5) Let $N$ and $T$ be as above. For $t \in J_{T} \backslash(\Gamma \cup\{0\})$ and $\sigma \in\left[\sigma_{1}, \sigma_{2}\right]$, $\sigma+\sqrt{-1} t \in G \quad$ and $\quad\left|\log \zeta(\sigma+\sqrt{-1} t)-\log \zeta_{N}(\sigma+\sqrt{-1} t)\right| \leq \frac{\eta^{1 / 20}}{1-\eta^{1 / 20}}$.

$\odot$ First, on $G \cap\{s \in \mathbb{C} ; \operatorname{Re} s>0\}$,

$$
\begin{gathered}
\log \zeta(s)=\log \frac{\pi^{2}}{6}+\int_{2}^{s} \frac{\zeta^{\prime}(z)}{\zeta(z)} d z \\
\log \zeta_{N}(s)=\log \zeta_{N}(2)+\int_{2}^{s} \frac{\zeta_{N}^{\prime}(z)}{\zeta_{N}(z)} d z .
\end{gathered}
$$

Since $\zeta(s)=\left(f_{N}(s)+1\right) \zeta_{N}(s)$,

$$
\begin{aligned}
\frac{\zeta^{\prime}(s)}{\zeta(s)}-\frac{\zeta_{N}^{\prime}(s)}{\zeta_{N}(s)} & =\frac{f_{N}^{\prime}(s) \zeta_{N}(s)+\left(f_{N}(s)+1\right) \zeta_{N}^{\prime}(s)}{\left(f_{N}(s)+1\right) \zeta_{N}(s)}-\frac{\zeta_{N}^{\prime}(s)}{\zeta_{N}(s)} \\
& =\frac{f_{N}^{\prime}(s)}{f_{N}(s)+1}
\end{aligned}
$$

and thus

$$
\log \zeta(s)-\log \zeta_{N}(s)=\log \zeta(2)-\log \zeta_{N}(2)+\int_{2}^{s} \frac{f_{N}^{\prime}(z)}{f_{N}(z)+1} d z
$$


Now let $t \in J_{T} \backslash(\Gamma \cup\{0\})$, and let $\sigma \in\left[\sigma_{1}, \sigma_{2}\right]$. By the definition of $\Gamma, \sigma^{\prime}+$ $\sqrt{-1} t \in G\left(\forall \sigma^{\prime} \in \mathbb{R}\right)$. By the definition of $G$,

$$
0<\exists \varepsilon<\delta \quad \text { s.t. }\left\{\sigma^{\prime}+\sqrt{-1} t^{\prime} ;\left|t^{\prime}-t\right|<\varepsilon\right\} \subset G .
$$

From the continuity of $f_{N}$ on $G \cap\{s \in \mathbb{C} ; \operatorname{Re} s>0\}$ and (4),

$$
0<\exists \varepsilon^{\prime}<\varepsilon \quad \text { s.t. }\left\{\begin{array}{c}
\left|f_{N}\left(\sigma^{\prime}+\sqrt{-1} t^{\prime}\right)\right|<\eta^{1 / 20}<1 \\
\text { for } \sigma_{1}-\varepsilon^{\prime}<\sigma^{\prime}<\sigma_{2}+\varepsilon^{\prime} \text { and }\left|t^{\prime}-t\right|<\varepsilon^{\prime} .
\end{array}\right.
$$

For $\sigma^{\prime}>\frac{3}{2}$ and $t^{\prime} \in \mathbb{R}$,

$$
\begin{aligned}
\left|f_{N}\left(\sigma^{\prime}+\sqrt{-1} t^{\prime}\right)\right| & =\left|\sum_{\substack{n \geq 2 ; \\
p_{1} \nmid n, \ldots, p_{N} \nmid n}} \frac{1}{n^{\sigma^{\prime}+\sqrt{-1} t^{\prime}}}\right|[\odot(4.7)] \\
& \leq \sum_{\substack{n \geq 2 ; \\
p_{1} \nmid n, \ldots, p_{N} \nmid n}} \frac{1}{n^{\sigma^{\prime}}} \\
& \leq \sum_{n>p_{N}} \frac{1}{n^{\sigma^{\prime}}} \\
& \leq \sum_{n>p_{N_{0}}} \frac{1}{n^{2 \sigma_{0}}}\left[\odot \sigma^{\prime}>\frac{3}{2}>2 \sigma_{0}\right] \\
& <\frac{\eta}{2}[\odot(4.9)] \\
& <\frac{1}{2} .
\end{aligned}
$$

Therefore, if we let

$$
\begin{aligned}
G_{0} & :=\left\{\sigma^{\prime}+\sqrt{-1} t^{\prime} ; \sigma^{\prime}>\sigma_{1}-\varepsilon^{\prime},\left|t^{\prime}-t\right|<\varepsilon^{\prime}\right\} \cup\left\{\sigma^{\prime}+\sqrt{-1} t^{\prime} ; \sigma^{\prime}>\frac{3}{2}\right\} \\
& \subset G \cap\{s ; \operatorname{Re} s>0\},
\end{aligned}
$$

$G_{0}$ is a simply connected domain of $\mathbb{C}$ and $\left|f_{N}(s)\right|<1$ on $G_{0}$, so that $\log \left(f_{N}(s)+\right.$ $1):=\int_{1}^{f_{N}(s)+1} w^{-1} d w$ can be defined on $G_{0}$ (see (2.4)), and

$$
(\log (f(s)+1))^{\prime}=\frac{f_{N}^{\prime}(s)}{f_{N}(s)+1}
$$

holds. Since $\sigma+\sqrt{-1} t, 2 \in G_{0}$,

$$
\begin{aligned}
\int_{2}^{\sigma+\sqrt{-1} t} \frac{f_{N}^{\prime}(s)}{f_{N}(s)+1} d s & =\left[\log \left(f_{N}(s)+1\right)\right]_{2}^{\sigma+\sqrt{-1} t} \\
& =\left.\log \left(f_{N}(s)+1\right)\right|_{s=\sigma+\sqrt{-1} t}-\log \left(f_{N}(2)+1\right) .
\end{aligned}
$$

Noting that

$$
\log \left(f_{N}(2)+1\right)=\log \frac{\zeta(2)}{\zeta_{N}(2)}=\log \zeta(2)-\log \zeta_{N}(2)
$$




$$
\begin{aligned}
|\log (w+1)| & \leq \frac{|w|}{1-|w|} \quad(|w|<1), \\
\left|f_{N}(\sigma+\sqrt{-1} t)\right| & <\eta^{1 / 20}<1,
\end{aligned}
$$

we have by (4.10) that

$$
\begin{aligned}
& \left|\log \zeta(\sigma+\sqrt{-1} t)-\log \zeta_{N}(\sigma+\sqrt{-1} t)\right| \\
& \quad=\left|\log \zeta(2)-\log \zeta_{N}(2)+\log \left(f_{N}(s)+1\right)\right|_{s=\sigma+\sqrt{-1} t}-\log \zeta(2)+\log \zeta_{N}(2) \mid \\
& \quad \leq\left.\frac{\left|f_{N}(s)\right|}{1-\left|f_{N}(s)\right|}\right|_{s=\sigma+\sqrt{-1} t} \\
& \quad<\frac{\eta^{1 / 20}}{1-\eta^{1 / 20}} .
\end{aligned}
$$

(6) For each $\varepsilon>0$,

$$
\begin{aligned}
& P\left(\sup _{\sigma_{1} \leq \sigma \leq \sigma_{2}}\left|\widetilde{X}_{T}(\sigma)-\widetilde{X}_{T, N}(\sigma)\right| \geq \varepsilon\right) \\
& \quad \leq \mathbf{1}_{\frac{\eta^{1 / 20}}{1-\eta^{1 / 20}} \geq \varepsilon}+1-\frac{\lfloor T\rfloor-1-\sqrt{\eta} T}{T}\left(1-2\left(\frac{\sigma_{3}-\sigma_{0}}{\pi}\right)^{1 / 2} \eta^{1 / 5}\right) .
\end{aligned}
$$

Here $N \geq N_{0}\left(\sigma_{0}, \eta\right)$ and $T \geq T_{0}\left(N, \sigma_{1}, \sigma_{2}, \eta\right)$.

$\odot$ Fix $\varepsilon>0$. This estimate is obtained in the following way:

$$
\begin{aligned}
P\left(\sup _{\sigma_{1} \leq \sigma \leq \sigma_{2}}\left|\tilde{X}_{T}(\sigma)-\widetilde{X}_{T, N}(\sigma)\right| \geq \varepsilon\right) & =P\left(\sup _{\sigma_{1} \leq \sigma \leq \sigma_{2}} \mid \mathbf{1}_{G}\left(\sigma+\sqrt{-1} U_{T}\right) \log \zeta\left(\sigma+\sqrt{-1} U_{T}\right)\right. \\
& \left.-\log \zeta_{N}\left(\sigma+\sqrt{-1} U_{T}\right) \mid \geq \varepsilon\right) \quad(\text { see }(1.3) \text { and }(4.6)) \\
= & P\left(U_{T} \notin \Gamma \cup\{0\}, \sup _{\sigma_{1} \leq \sigma \leq \sigma_{2}} \mid \log \zeta\left(\sigma+\sqrt{-1} U_{T}\right)\right. \\
& \left.-\log \zeta_{N}\left(\sigma+\sqrt{-1} U_{T}\right) \mid \geq \varepsilon\right) \\
& {\left[\odot U_{T} \notin \Gamma \cup\{0\} P-\text { almost surely, and then } \sigma+\sqrt{-1} U_{T} \in G(\forall \sigma \in \mathbb{R})\right] } \\
= & \frac{1}{2 T} \mu\left([-T, T] \cap(\Gamma \cup\{0\})^{\complement}\right. \\
& \left.\cap\left\{\sup _{\sigma_{1} \leq \sigma \leq \sigma_{2}}\left|\log \zeta(\sigma+\sqrt{-1} t)-\log \zeta_{N}(\sigma+\sqrt{-1} t)\right| \geq \varepsilon\right\}\right) \\
& {\left[\odot U_{T} \text { is uniformly distributed on }[-T, T]\right] } \\
= & \frac{1}{2 T} \mu\left(J_{T} \cap(\Gamma \cup\{0\})^{\complement}\right. \\
& \left.\cap\left\{\sup _{\sigma_{1} \leq \sigma \leq \sigma_{2}}\left|\log \zeta(\sigma+\sqrt{-1} t)-\log \zeta_{N}(\sigma+\sqrt{-1} t)\right| \geq \varepsilon\right\}\right) \\
& +\frac{1}{2 T} \mu\left(\left(-J_{T}\right) \cap(\Gamma \cup\{0\})^{\complement}\right.
\end{aligned}
$$




$$
\begin{aligned}
& \left.\cap\left\{\sup _{\sigma_{1} \leq \sigma \leq \sigma_{2}}\left|\log \zeta(\sigma+\sqrt{-1} t)-\log \zeta_{N}(\sigma+\sqrt{-1} t)\right| \geq \varepsilon\right\}\right) \\
& +\frac{1}{2 T} \mu\left([-T, T] \cap\left(J_{T} \cup\left(-J_{T}\right)\right)^{\complement} \cap(\Gamma \cup\{0\})^{\complement}\right. \\
& \left.\cap\left\{\sup _{\sigma_{1} \leq \sigma \leq \sigma_{2}}\left|\log \zeta(\sigma+\sqrt{-1} t)-\log \zeta_{N}(\sigma+\sqrt{-1} t)\right| \geq \varepsilon\right\}\right) \\
& =\frac{1}{T} \mu\left(J_{T} \cap(\Gamma \cup\{0\})^{\complement}\right. \\
& \left.\cap\left\{\sup _{\sigma_{1} \leq \sigma \leq \sigma_{2}}\left|\log \zeta(\sigma+\sqrt{-1} t)-\log \zeta_{N}(\sigma+\sqrt{-1} t)\right| \geq \varepsilon\right\}\right) \\
& +\frac{1}{2 T} \mu\left([-T, T] \cap\left(J_{T} \cup\left(-J_{T}\right)\right)^{\complement} \cap(\Gamma \cup\{0\})^{\complement}\right. \\
& \left.\cap\left\{\sup _{\sigma_{1} \leq \sigma \leq \sigma_{2}}\left|\log \zeta(\sigma+\sqrt{-1} t)-\log \zeta_{N}(\sigma+\sqrt{-1} t)\right| \geq \varepsilon\right\}\right) \\
& \lceil\odot \text { Since }-(\Gamma \cup\{0\})=\Gamma \cup\{0\} \text {, } \\
& t \in\left(-J_{T}\right) \cap(\Gamma \cup\{0\})^{\complement} \Leftrightarrow-t \in J_{T} \cap(\Gamma \cup\{0\})^{\complement} . \\
& \text { AAlso } \log \zeta(\bar{s})=\overline{\log \zeta(s)}(s \in G) \text { and } \log \zeta_{N}(\bar{s})=\overline{\log \zeta_{N}(s)}(s ; \operatorname{Re} s>0) \text { ] } \\
& \leq \mathbf{1}_{\frac{\eta^{1 / 20}}{1-\eta^{1 / 20}} \geq \varepsilon}+\frac{1}{2 T} \mu\left([-T, T] \cap\left(J_{T} \cup\left(-J_{T}\right)\right)^{\complement}\right) \\
& =1_{\frac{\eta^{1 / 20}}{1-\eta^{1 / 20}} \geq \varepsilon}+1-\frac{1}{T} \mu\left(J_{T}\right) \\
& \leq \mathbf{1}_{\frac{\eta^{1 / 20}}{1-\eta^{1 / 20}} \geq \varepsilon}+1-\frac{\lfloor T\rfloor-1-\sqrt{\eta} T}{T}(1-2 \delta) \quad[\odot(3)] \\
& =1_{\frac{\eta^{1 / 20}}{1-\eta^{1 / 20}} \geq \varepsilon}+1-\frac{\lfloor T\rfloor-1-\sqrt{\eta} T}{T}\left(1-2\left(\frac{\sigma_{3}-\sigma_{0}}{\pi}\right)^{1 / 2} \eta^{1 / 5}\right) .
\end{aligned}
$$

(7) In (6), letting $T \rightarrow \infty$ and then $N \rightarrow \infty$, we have

$$
\begin{aligned}
& \limsup _{N \rightarrow \infty} \limsup _{T \rightarrow \infty} P\left(\sup _{\sigma_{1} \leq \sigma \leq \sigma_{2}}\left|\widetilde{X}_{T}(\sigma)-\tilde{X}_{T, N}(\sigma)\right| \geq \varepsilon\right) \\
& \quad \leq \mathbf{1}_{\frac{\eta^{1 / 20}}{1-\eta^{1 / 20}} \geq \varepsilon}+1-(1-\sqrt{\eta})\left(1-2\left(\frac{\sigma_{3}-\sigma_{0}}{\pi}\right)^{1 / 2} \eta^{1 / 5}\right) \\
& \quad \rightarrow 0 \text { as } \eta \searrow 0,
\end{aligned}
$$

which is the assertion of Step 1.

\section{STEP 2}

For each $N \in \mathbb{N}$,

$$
\begin{aligned}
& \text { the distribution of }\left\{\widetilde{X}_{T, N}(\sigma)\right\}_{\sigma>1 / 2} \\
& \rightarrow \text { the distribution of }\left\{\sum_{i=1}^{N}-\log \left(1-\frac{e\left(-\log p_{i}\right)}{p_{i}^{\sigma}}\right)\right\}_{\sigma>1 / 2} \text { as } T \rightarrow \infty .
\end{aligned}
$$


Namely, for $\frac{1}{2}<\sigma_{1}<\sigma_{2}<\infty$ and $\forall \Phi: C\left(\left[\sigma_{1}, \sigma_{2}\right] \rightarrow \mathbb{C}\right) \rightarrow \mathbb{R}$ that are bounded continuous,

$$
\lim _{T \rightarrow \infty} E\left[\Phi\left(\widetilde{X}_{T, N}(\cdot)\right)\right]=E^{\mathbf{P}}\left[\Phi\left(\sum_{i=1}^{N}-\log \left(1-\frac{e\left(-\log p_{i}\right)}{p_{i}^{\cdot}}\right)\right)\right] .
$$

Proof

First, by (4.6) and (4.5)

$$
\widetilde{X}_{T, N}(\sigma)=\sum_{i=1}^{N}-\log \left(1-\frac{e^{\sqrt{-1}\left(-\log p_{i}\right) U_{T}}}{p_{i}^{\sigma}}\right)
$$

Next, by Proposition 2.1,

$$
\text { the distribution of }\left(e^{\sqrt{-1}\left(-\log p_{i}\right) U_{T}}\right)_{i=1}^{N}
$$

$$
\rightarrow \text { the distribution of }\left(e\left(-\log p_{i}\right)\right)_{i=1}^{N} \quad \text { as } T \rightarrow \infty .
$$

For, since

the distribution of $\left(\cos \left(-\log p_{1}\right) U_{T}, \sin \left(-\log p_{1}\right) U_{T}\right.$,

$$
\begin{aligned}
& \left.\ldots, \cos \left(-\log p_{N}\right) U_{T}, \sin \left(-\log p_{N}\right) U_{T}\right) \\
& =P_{T}^{\left(\cos \left(-\log p_{1}\right) \cdot \sin \left(-\log p_{1}\right) \cdot, \ldots, \cos \left(-\log p_{N}\right) \cdot \sin \left(-\log p_{N}\right) \cdot\right)} \quad(\text { see }(4.3)) \\
& \rightarrow \mathbf{P}_{\circ} \pi_{\left(\cos \left(-\log p_{1}\right) \cdot, \sin \left(-\log p_{1}\right) \cdot, \ldots, \cos \left(-\log p_{N}\right) \cdot \sin \left(-\log p_{N}\right) \cdot\right)}^{-1} \quad \text { as } T \rightarrow \infty
\end{aligned}
$$

we have that for any bounded continuous function $F: \mathbb{C}^{N} \rightarrow \mathbb{R}$

$$
\begin{aligned}
E[ & \left.F\left(e^{\sqrt{-1}\left(-\log p_{1}\right) U_{T}}, \ldots, e^{\sqrt{-1}\left(-\log p_{N}\right) U_{T}}\right)\right] \\
= & \int_{\mathbb{R}^{2 N}} F\left(a_{1}+\sqrt{-1} b_{1}, \ldots, a_{N}+\sqrt{-1} b_{N}\right) \\
& P_{T}^{\left(\cos \left(-\log p_{1}\right) \cdot, \sin \left(-\log p_{1}\right) \cdot, \ldots, \cos \left(-\log p_{N}\right) \cdot \sin \left(-\log p_{N}\right) \cdot\right)}\left(d a_{1} d b_{1} \cdots d a_{N} d b_{N}\right) \\
& \rightarrow \int_{\mathbb{R}^{2 N}} F\left(a_{1}+\sqrt{-1} b_{1}, \ldots, a_{N}+\sqrt{-1} b_{N}\right) \\
& \mathbf{P} \circ \pi_{\left(\cos \left(-\log p_{1}\right) \cdot \sin \left(-\log p_{1}\right) \cdot, \ldots, \cos \left(-\log p_{N}\right) \cdot \sin \left(-\log p_{N}\right) \cdot\right)}^{-1}\left(d a_{1} d b_{1} \cdots d a_{N} d b_{N}\right) \\
= & E^{\mathbf{P}}\left[F\left(e\left(-\log p_{1}\right), \ldots, e\left(-\log p_{N}\right)\right)\right] \quad \text { as } T \rightarrow \infty .
\end{aligned}
$$

This shows (4.12).

The assertion of Step 2 is obvious from (4.11) and (4.12).

\section{STEP 3}

The following convergence holds $\mathbf{P}$-almost surely. As $N \rightarrow \infty$,

$$
\sum_{i=1}^{N}-\log \left(1-\frac{e\left(-\log p_{i}\right)}{p_{i}^{\sigma}}\right) \rightrightarrows \tilde{X}(\sigma) \quad \text { on }\left[\sigma_{0}, \infty\right), \forall \sigma_{0}>\frac{1}{2}
$$


Proof

For this, see the proof of Theorem 2.1.

STEP 4

For $\frac{1}{2}<\sigma_{1}<\sigma_{2}<\infty$ and $\forall \Phi: C\left(\left[\sigma_{1}, \sigma_{2}\right] \rightarrow \mathbb{C}\right) \rightarrow \mathbb{R}$ that are bounded uniformly continuous,

$$
\lim _{T \rightarrow \infty} E\left[\Phi\left(\widetilde{X}_{T}(\cdot)\right)\right]=E^{\mathbf{P}}[\Phi(\widetilde{X}(\cdot))]
$$

Thus, by Claim 3.2, $P \circ \widetilde{X}_{T}^{-1} \rightarrow \mathbf{P} \circ \widetilde{X}^{-1}$ as $T \rightarrow \infty$.

Proof

Let $\frac{1}{2}<\sigma_{1}<\sigma_{2}<\infty$, and let $\Phi: C\left(\left[\sigma_{1}, \sigma_{2}\right] \rightarrow \mathbb{C}\right) \rightarrow \mathbb{R}$ be bounded uniformly continuous. Then, $\forall \rho>0$, take $\varepsilon>0$ so that

$$
\sup _{\sigma_{1} \leq \sigma \leq \sigma_{2}}|w(\sigma)-z(\sigma)|<\varepsilon \Rightarrow|\Phi(w)-\Phi(z)|<\rho .
$$

Then

$$
\begin{aligned}
&\left|E\left[\Phi\left(\widetilde{X}_{T}(\cdot)\right)\right]-E^{\mathbf{P}}[\Phi(\widetilde{X}(\cdot))]\right| \\
& \leq\left|E\left[\Phi\left(\widetilde{X}_{T}(\cdot)\right)\right]-E\left[\Phi\left(\widetilde{X}_{T, N}(\cdot)\right)\right]\right|+\left|E\left[\Phi\left(\widetilde{X}_{T, N}(\cdot)\right)\right]-E^{\mathbf{P}}[\Phi(\widetilde{X}(\cdot))]\right| \\
& \leq E\left[\left|\Phi\left(\widetilde{X}_{T}(\cdot)\right)-\Phi\left(\widetilde{X}_{T, N}(\cdot)\right)\right|\right]+\left|E\left[\Phi\left(\widetilde{X}_{T, N}(\cdot)\right)\right]-E^{\mathbf{P}}[\Phi(\widetilde{X}(\cdot))]\right| \\
&= E\left[\left|\Phi\left(\widetilde{X}_{T}(\cdot)\right)-\Phi\left(\widetilde{X}_{T, N}(\cdot)\right)\right| ; \sup _{\sigma_{1} \leq \sigma \leq \sigma_{2}}\left|\widetilde{X}_{T}(\sigma)-\widetilde{X}_{T, N}(\sigma)\right|<\varepsilon\right] \\
&+E\left[\left|\Phi\left(\widetilde{X}_{T}(\cdot)\right)-\Phi\left(\widetilde{X}_{T, N}(\cdot)\right)\right| ; \sup _{\sigma_{1} \leq \sigma \leq \sigma_{2}}\left|\widetilde{X}_{T}(\sigma)-\widetilde{X}_{T, N}(\sigma)\right| \geq \varepsilon\right] \\
&+\left|E\left[\Phi\left(\widetilde{X}_{T, N}(\cdot)\right)\right]-E^{\mathbf{P}}[\Phi(\widetilde{X}(\cdot))]\right| \\
& \leq \rho+2\|\Phi\|_{\infty} P\left(\sup _{\sigma_{1} \leq \sigma \leq \sigma_{2}}\left|\widetilde{X}_{T}(\sigma)-\widetilde{X}_{T, N}(\sigma)\right| \geq \varepsilon\right) \\
&+\left|E\left[\Phi\left(\widetilde{X}_{T, N}(\cdot)\right)\right]-E^{\mathbf{P}}[\Phi(\widetilde{X}(\cdot))]\right|,
\end{aligned}
$$

where $\|\Phi\|_{\infty}=\sup _{w \in C\left(\left[\sigma_{1}, \sigma_{2}\right] \rightarrow \mathbb{C}\right)}|\Phi(w)|$. Letting $T \rightarrow \infty$, we have by Step 2 that

$$
\begin{aligned}
\limsup _{T \rightarrow \infty}\left|E\left[\Phi\left(\widetilde{X}_{T}(\cdot)\right)\right]-E^{\mathbf{P}}[\Phi(\widetilde{X}(\cdot))]\right| \\
\leq \rho+2\|\Phi\|_{\infty} \limsup _{T \rightarrow \infty} P\left(\sup _{\sigma_{1} \leq \sigma \leq \sigma_{2}}\left|\widetilde{X}_{T}(\sigma)-\widetilde{X}_{T, N}(\sigma)\right| \geq \varepsilon\right) \\
\quad+\left|E^{\mathbf{P}}\left[\Phi\left(\sum_{i=1}^{N}-\log \left(1-\frac{e\left(-\log p_{i}\right)}{p_{i}}\right)\right)\right]-E^{\mathbf{P}}[\Phi(\tilde{X}(\cdot))]\right|
\end{aligned}
$$

By Steps 1 and 3, the right-hand side $\rightarrow \rho$ as $N \rightarrow \infty$. Thus, letting $\rho \searrow 0$, we obtain the convergence of Step 4. 


\section{COROLLARY 4.1}

The distribution of a continuous process $\left\{\zeta\left(\sigma+\sqrt{-1} U_{T}\right)\right\}_{\sigma>1 / 2}$ is weakly convergent to that of a continuous process $\left\{e^{\widetilde{X}(\sigma)}=\prod_{p}\left(1-(e(-\log p)) / p^{\sigma}\right)^{-1}\right\}_{\sigma>1 / 2}$ as $T \rightarrow \infty$.

Proof

Note that $U_{T} \notin \Gamma \cup\{0\}$ almost surely. This tells us that, with probability 1, $\sigma+\sqrt{-1} U_{T} \in G$ and so $e^{\widetilde{X}_{T}(\sigma)}=\zeta\left(\sigma+\sqrt{-1} U_{T}\right)(\forall \sigma \in \mathbb{R})$. From Theorem 4.1, the assertion of the corollary follows at once.

\section{References}

[1] H. Bohr, Zur Theorie der Riemann'schen Zetafunktion im Kritischen Streifen (in German), Acta Math. 40 (1915), 67-100.

[2] H. Bohr and R. Courant, Neue Anwendungen der Theorie der Diophantischen Approximationen auf die Riemannsche Zetafunktion (in German), J. Reine Angew. Math. 144 (1914), 249-274.

[3] H. Bohr and B. Jessen, Über die Wertverteilung der Riemannschen Zetafunktion, Erste Mitteilung (in German), Acta Math. 54 (1930), 1-35; Zweite Mitteilung (in German), Acta Math. 58 (1932), 1-55. MR 1555301; MR 1555343. DOI 10.1007/BF02547516; DOI 10.1007/BF02547773.

[4] V. Borchsenius and B. Jessen, Mean motions and values of the Riemann zeta function, Acta Math. 80 (1948), 97-166. MR 0027796.

[5] K. Itô, Introduction to Probability Theory, Cambridge Univ. Press, Cambridge, 1984. MR 0777504.

[6] B. Jessen and A. Wintner, Distribution functions and the Riemann zeta function, Trans. Amer. Math. Soc., 38 (1935), no. 1, 48-88. MR 1501802. DOI 10.2307/1989728.

[7] Y. Kawada, Number Theory: From Classical Number Theory to Class Field Theory (in Japanese), Iwanami, Tokyo, 2010.

[8] S. Kotani, Measure and Probability (in Japanese), Iwanami, Tokyo, 2005.

[9] A. Laurinchikas, A limit theorem for Dirichlet L-series (in Russian), Mat. Zametki 25 (1979), 481-485, 635; English translation in Math. Notes 25 (1979), 251-253. MR 0534290.

[10] - "Limit theorems for the Riemann zeta-function in the complex space" in Probability Theory and Mathematical Statistics, II (Vilnius, 1989), Mokslas, Vilnius, Lithuania, 1990, 59-69. MR 1153860.

[11] Limit Theorems for the Riemann Zeta-Function, Kluwer, Dordrecht, 1996.

[12] K. Matsumoto, "Value-distribution of zeta-functions" in Analytic Number Theory (Tokyo, 1988), Lecture Notes in Math. 1434, Springer, Berlin, 1990, 178-187. MR 1071754. DOI 10.1007/BFb0097134. 
[13] _ Asymptotic probability measures of zeta-functions of algebraic number fields, J. Number Theory 40 (1992), 187-210. MR 1149737.

DOI 10.1016/0022-314X(92)90039-R.

[14] _ Riemann Zeta Function (in Japanese), Asakura, Tokyo, 2005.

[15] T. Murayama and S. Takanobu, The behavior of the limit distribution in Bohr-Jessen limit theorem as $\sigma \searrow \frac{1}{2}$ - asymptotic expansion of probability densities (in Japanese), lecture at "MSJ Spring Meeting 2011," Tokyo, 2011.

[16] H. Sato, From Measure to Probability - Introduction to Probability Theory (in Japanese), Kyoritsu, Tokyo, 1994.

[17] D. W. Stroock, Probability Theory: An Analytic View, rev. ed., Cambridge Univ. Press, Cambridge, 1999. MR 2760872.

[18] S. Takanobu, Bohr-Jessen Limit Theorem, Revisited, Math. Soc. Japan Mem. 31, Math. Soc. Japan, Tokyo, 2013.

[19] G. Tenenbaum, Introduction to Analytic and Probabilistic Number Theory, Cambridge Stud. Adv. Math. 46, Cambridge Univ. Press, Cambridge, 1995. MR 1342300.

Faculty of Mathematics and Physics, Institute of Science and Engineering, Kanazawa University, Kakuma-machi, Kanazawa 920-1192, Japan;

takanob@staff.kanazawa-u.ac.jp 